\title{
AVOIDING SHARED CLOCKS IN NETWORKS OF TIMED AUTOMATA*
}

\author{
SANDIE BALAGUER $^{a}$ AND THOMAS CHATAIN $^{b}$
}

LSV (ENS Cachan, CNRS, Inria), 61, avenue du Président Wilson, 94235 CACHAN Cedex, France

e-mail address: \{balaguer,chatain\}@lsv.ens-cachan.fr

\begin{abstract}
Networks of timed automata (NTA) are widely used to model distributed real-time systems. Quite often in the literature, the automata are allowed to share clocks, i.e. transitions of one automaton may be guarded by conditions on the value of clocks reset by another automaton. This is a problem when one considers implementing such model in a distributed architecture, since reading clocks a priori requires communications which are not explicitly described in the model. We focus on the following question: given an NTA $A_{1} \| A_{2}$ where $A_{2}$ reads some clocks reset by $A_{1}$, does there exist an NTA $A_{1}^{\prime} \| A_{2}^{\prime}$ without shared clocks with the same behavior as the initial NTA? For this, we allow the automata to exchange information during synchronizations only, in particular by copying the value of their neighbor's clocks. We discuss a formalization of the problem and define an appropriate behavioural equivalence. Then we give a criterion using the notion of contextual timed transition system, which represents the behavior of $A_{2}$ when in parallel with $A_{1}$. Finally, we effectively build $A_{1}^{\prime} \| A_{2}^{\prime}$ when it exists.
\end{abstract}

\section{INTRODUCTION}

Timed automata AD94 are one of the most famous formal models for real-time systems. They have been deeply studied and very mature tools are available, like UPPAAL [BDL04, EPSILON [CGL93] and Kronos [BDM ${ }^{+} 98$ ].

Networks of Timed Automata (NTA) are a natural generalization to model real-time distributed systems. In this formalism, each automaton has a set of clocks that constrain its real-time behavior. But quite often in the literature, the automata are allowed to share clocks, which provides a special way of making the behavior of one automaton depend on what the others do. Actually shared clocks are relatively well accepted and can be a convenient feature for modeling systems. Imagine for instance several agents performing together a distributed task according to a predefined schedule. In a typical implementation

2012 ACM CCS: [Theory of computation]: Models of computation-Timed and hybrid models; Models of computation-Concurrency-Distributed computing models.

Key words and phrases: networks of timed automata, shared clocks, implementation on distributed architecture, contextual timed transition system, behavioral equivalence for distributed systems.

* This paper extends the version presented at CONCUR'12 [BC12].

${ }^{a}$ This work is partially supported by the French ANR project ImpRo. 
the schedule would be sent to the agents at the beginning and every agent would store its own copy of the schedule. But for a (simplified) model of the system, it may be easier to have one timed automaton modeling a single copy of the schedule and every agent referring to it via shared clocks.

Since NTA are almost always given a sequential semantics, shared clocks can be handled very easily even by tools: once the NTA is transformed into a single timed automaton by the classical product construction, the notion of distribution is lost and the notion of shared clock itself becomes meaningless. Nevertheless, implementing a model with shared clocks in a multi-core architecture is not straightforward since reading clocks a priori requires communications which are not explicitly described in the model.

Here we are concerned with the expressive power of shared clocks according to the distributed nature of the system. We are aware of only one previous study about this aspect, presented in [LPW07]. Our purpose is to identify NTA where sharing clocks could be avoided, i.e. NTA which syntactically use shared clocks, but whose semantics could be achieved by another NTA without shared clocks. For simplicity, we look at NTA made of two automata $A_{1}$ and $A_{2}$ where only $A_{2}$ reads clocks reset by $A_{1}$. The first step is to formalize which aspect of the semantics we want to preserve in this setting. Then the idea is essentially to detect cases where $A_{2}$ can avoid reading a clock because its value does not depend on the actions that are local to $A_{1}$ and thus unobservable to $A_{2}$. To generalize this idea we have to compute the knowledge of $A_{2}$ about the state of $A_{1}$. We show that this knowledge is maximized if we allow $A_{1}$ to communicate its state to $A_{2}$ each time they synchronize on a common action.

In order to formalize our problem we need an appropriate notion of behavioral equivalence between two NTA. We explain why classical comparisons based on the sequential semantics, like timed bisimulation, are not sufficient here. We need a notion that takes the distributed nature of the system into account. That is, a component cannot observe the moves and the state of the other and must choose its local actions according to its partial knowledge of the state of the system. We define the notion of contextual timed transition systems (contextual TTS) in order to formalize this idea.

Then we express the problem of avoiding shared clocks in terms of contextual TTS and we give a characterization of the NTA for which shared clocks can be avoided. Finally we effectively construct an NTA without shared clocks with the same behavior as the initial one, when it exists. A possible interest is to allow a designer to use shared clocks as a high-level feature in a model of a protocol, and rely on our transformation to make it implementable.

Related work. The semantics of time in distributed systems has already been debated. The idea of localizing clocks has already been proposed and some authors $\mathrm{ABG}^{+}$08, DL07, BJLY98 have even suggested to use local-time semantics with independently evolving clocks. Here we stay in the classical setting of perfect clocks evolving at the same speed. This is a key assumption that provides an implicit synchronization and lets us know some clock values without reading them.

Many formalisms exist for real-time distributed systems, among which NTA [AD94] and time Petri nets [Mer74]. So far, their expressiveness was compared $\mathrm{BCH}^{+} 05$, BR08, CR06, Srb08] essentially in terms of sequential semantics that forget concurrency. In [BCH12, we defined a concurrency-preserving translation from time Petri nets to networks of timed automata. This transformation uses shared clocks and the question whether these could be avoided remained open. 
While partial-order semantics and unfoldings are well known for untimed systems, they have been very little studied for distributed real-time systems [CCJ06, BHR06. Partial order reductions for (N)TA were proposed in [Min99, BJLY98, LNZ05. Behavioral equivalence relations for distributed systems, like history-preserving bisimulations, were defined for untimed systems only BDKP91, vGG01].

Finally, our notion of contextual TTS deals with knowledge of agents in distributed systems. This is the aim of epistemic logics HFMV95, which have been extended to real-time in WL04, Dim09]. Our notion of contextual TTS also resembles the technique of partitioning states based on observation, used in timed games with partial observability BDMP03, DLLN09].

Organization of the paper. The paper is organized as follows. Section 1 recalls basic notions about TTS and NTA. Section 2 presents the problem of avoiding shared clocks on examples and rises the problem of comparing NTA component by component. For this, the notion of contextual TTS is developed in Section 3. The problem of avoiding shared clocks is formalized and characterized in terms of contextual TTS. Then Section 4 presents our construction.

\section{Preliminaries}

1.1. Timed Transition Systems. The behavior of timed systems is often described as timed transition systems.

Definition 1.1. A timed transition system (TTS) is a tuple $\left(S, s_{0}, \Sigma, \rightarrow\right)$ where $S$ is a set of states, $s_{0} \in S$ is the initial state, $\Sigma$ is a finite set of actions disjoint from $\mathbb{R}_{\geq 0}$, and $\rightarrow \subseteq S \times\left(\Sigma \cup \mathbb{R}_{\geq 0}\right) \times S$ is a set of edges.

For any $a \in \Sigma \cup \mathbb{R}_{\geq 0}$, we write $s \stackrel{a}{\rightarrow} s^{\prime}$ if $\left(s, a, s^{\prime}\right) \in \rightarrow$, and $s \stackrel{a}{\rightarrow}$ if for some $s^{\prime}$, $\left(s, a, s^{\prime}\right) \in \rightarrow$. We define the transition relation $\Rightarrow$ as:

- $s \stackrel{\varepsilon}{\Rightarrow} s^{\prime}$ if $s(\stackrel{\varepsilon}{\rightarrow})^{*} s^{\prime}$

- $\forall a \in \Sigma, s \stackrel{a}{\Rightarrow} s^{\prime}$ if $s(\stackrel{\varepsilon}{\rightarrow})^{*} \stackrel{a}{\rightarrow}(\stackrel{\varepsilon}{\rightarrow})^{*} s^{\prime}$,

- $\forall d \in \mathbb{R}_{\geq 0}, s \stackrel{d}{\Rightarrow} s^{\prime}$ if $s(\stackrel{\varepsilon}{\rightarrow})^{*} \stackrel{d_{0}}{\Rightarrow}(\stackrel{\varepsilon}{\rightarrow})^{*} \cdots \stackrel{d_{n}}{\Longrightarrow}(\stackrel{\varepsilon}{\rightarrow})^{*} s^{\prime}$, where $\sum_{k=0}^{n} d_{k}=d$.

A path of a TTS is a possibly infinite sequence of transitions $\rho=s \stackrel{d_{0}}{\longrightarrow} s_{0}^{\prime} \stackrel{a_{0}}{\longrightarrow}$ $\cdots s_{n} \stackrel{d_{n}}{\longrightarrow} s_{n}^{\prime} \stackrel{a_{n}}{\longrightarrow} \cdots$, where, for all $i, d_{i} \in \mathbb{R}_{\geq 0}$ and $a_{i} \in \Sigma$. A path is initial if it starts in $s_{0}$. A path $\rho=s \stackrel{d_{0}}{\rightarrow} s_{0}^{\prime} \stackrel{a_{0}}{\longrightarrow} \cdots s_{n} \stackrel{d_{n}}{\longrightarrow} s_{n}^{\prime} \stackrel{a_{n}}{\longrightarrow} s_{n}^{\prime} \cdots$ generates a timed word $w=\left(a_{0}, t_{0}\right)\left(a_{1}, t_{1}\right) \ldots\left(a_{n}, t_{n}\right) \ldots$ where, for all $i, t_{i}=\sum_{k=0}^{i} d_{k}$. The duration of $w$ is $\delta(w)=\sup _{i} t_{i}$ and the untimed word of $w$ is $\lambda(w)=a_{0} a_{1} \ldots a_{n} \ldots \mathrm{TW}_{0}(\Sigma)$ denotes the set of finite timed words of duration 0 over $\Sigma$, i.e. $\operatorname{TW}_{0}(\Sigma)=\left\{w \mid \delta(w)=0 \wedge \lambda(w) \in \Sigma^{*}\right\}$. $\operatorname{Paths}(\Sigma, d)$ denotes the set of finite paths of duration $d$ over $\Sigma$. Lastly, we write $s \stackrel{w}{\rightarrow} s^{\prime}$ if there is a path from $s$ to $s^{\prime}$ that generates the timed word $w$.

In the sequel, we use the following notations: for $i \in\{1,2\}, T_{i}=\left(S_{i}, s_{i}^{0}, \Sigma_{i}, \rightarrow_{i}\right)$ is a TTS, and $\Sigma_{i}^{\nexists}=\Sigma_{i} \backslash\{\varepsilon\}$, where $\varepsilon$ is the silent action. 
Product of timed transitions systems. The product of $T_{1}$ and $T_{2}$, denoted by $T_{1} \otimes T_{2}$, is the TTS $\left(S_{1} \times S_{2},\left(s_{1}^{0}, s_{2}^{0}\right), \Sigma_{1} \cup \Sigma_{2}, \rightarrow\right)$, where $\rightarrow$ is defined as:

- $\left(s_{1}, s_{2}\right) \stackrel{a}{\rightarrow}\left(s_{1}^{\prime}, s_{2}\right)$ iff $s_{1} \stackrel{a}{\rightarrow}{ }_{1} s_{1}^{\prime}$, for any $a \in \Sigma_{1} \backslash \Sigma_{2}^{q}$,

- $\left(s_{1}, s_{2}\right) \stackrel{a}{\rightarrow}\left(s_{1}, s_{2}^{\prime}\right)$ iff $s_{2} \stackrel{a}{\rightarrow} 2 s_{2}^{\prime}$, for any $a \in \Sigma_{2} \backslash \Sigma_{1}^{q}$,

- $\left(s_{1}, s_{2}\right) \stackrel{a}{\rightarrow}\left(s_{1}^{\prime}, s_{2}^{\prime}\right)$ iff $s_{1} \stackrel{a}{\rightarrow} 1 s_{1}^{\prime}$ and $s_{2} \stackrel{a}{\rightarrow}_{2} s_{2}^{\prime}$, for any $a \in\left(\Sigma_{1}^{\ddagger} \cap \Sigma_{2}^{\ddagger}\right) \cup \mathbb{R}_{\geq 0}$.

Timed Bisimulations. Let $\mathcal{R}$ be a binary relation over $S_{1} \times S_{2}$. $\mathcal{R}$ is a strong (resp. weak) timed bisimulation relation between $T_{1}$ and $T_{2}$ if $s_{1}^{0} \mathcal{R} s_{2}^{0}$ and $s_{1} \mathcal{R} s_{2}$ implies that, for any $a \in \Sigma \cup \mathbb{R}_{\geq 0}$, if $s_{1} \stackrel{a}{\rightarrow}_{1} s_{1}^{\prime}$, then, for some $s_{2}^{\prime}, s_{2} \stackrel{a}{\rightarrow}_{2} s_{2}^{\prime}$ (resp. $s_{2} \stackrel{a}{\Rightarrow}{ }_{2} s_{2}^{\prime}$ ) and $s_{1}^{\prime} \mathcal{R} s_{2}^{\prime}$; and conversely, if $s_{2} \stackrel{a}{\rightarrow} 2 s_{2}^{\prime}$, then, for some $s_{1}^{\prime}, s_{1} \stackrel{a}{\rightarrow}_{1} s_{1}^{\prime}$ (resp. $s_{1} \stackrel{a}{\Rightarrow}_{1} s_{1}^{\prime}$ ) and $s_{1}^{\prime} \mathcal{R} s_{2}^{\prime}$.

We write $T_{1} \approx T_{2}$ (resp. $T_{1} \sim T_{2}$ ) when there is a strong (resp. weak) timed bisimulation between $T_{1}$ and $T_{2}$.

1.2. Networks of Timed Automata. The set $\mathcal{B}(X)$ of clock constraints over the set of clocks $X$ is defined by the grammar $g::=x \bowtie k \mid g \wedge g$, where $x \in X, k \in \mathbb{N}$ and $\bowtie \in\{<, \leq,=, \geq,>\}$. Invariants are clock constraints of the form $i::=x \leq k|x<k| i \wedge i$.

Definition 1.2. A network of timed automata (NTA) AD94 is a parallel composition of timed automata (TA) denoted as $A_{1}\|\cdots\| A_{n}$, with $A_{i}=\left(L_{i}, \ell_{i}^{0}, X_{i}, \Sigma_{i}, E_{i}\right.$, Inv $\left._{i}\right)$ where $L_{i}$ is a finite set of locations, $\ell_{i}^{0} \in L_{i}$ is the initial location, $X_{i}$ is a finite set of clocks, $\Sigma_{i}$ is a finite set of actions, $E_{i} \subseteq L_{i} \times \mathcal{B}\left(X_{i}\right) \times \Sigma_{i} \times 2^{X_{i}} \times L_{i}$ is a set of edges, and $\operatorname{Inv}_{i}: L_{i} \rightarrow \mathcal{B}\left(X_{i}\right)$ assigns invariants to locations.

If $\left(\ell, g, a, r, \ell^{\prime}\right) \in E_{i}$, we also write $\ell \stackrel{g, a, r}{\longrightarrow} \ell^{\prime}$. For such an edge, $g$ is the guard, $a$ the action and $r$ the set of clocks to reset. $C_{i} \subseteq X_{i}$ is the set of clocks reset by $A_{i}$ and for $i \neq j$, $C_{i} \cap C_{j}$ may not be empty.

Semantics. For simplicity, we give the semantics of a network of two TA $A_{1} \| A_{2}$. We denote by $\left(\left(\ell_{1}, \ell_{2}\right), v\right)$ a state of the NTA, where $\ell_{1}$ and $\ell_{2}$ are the current locations, and $v: X \rightarrow \mathbb{R}_{\geq 0}$, with $X=X_{1} \cup X_{2}$, is a clock valuation that maps each clock to its current value. A state is legal only if its valuation $v$ satisfies the invariants of the current locations, denoted by $v \models \operatorname{Inv}_{1}\left(\ell_{1}\right) \wedge \operatorname{Inv}_{2}\left(\ell_{2}\right)$. For each set of clocks $r \subseteq X$, the valuation $v[r]$ is defined by $v[r](x)=0$ if $x \in r$ and $v[r](x)=v(x)$ otherwise. For each $d \in \mathbb{R}_{>0}$, the valuation $v+d$ is defined by $(v+d)(x)=v(x)+d$ for each $x \in X$. Then, the TTS generated by $A_{1} \| A_{2}$ is $\operatorname{TTS}\left(A_{1} \| A_{2}\right)=\left(S, s_{0}, \Sigma_{1} \cup \Sigma_{2}, \rightarrow\right)$, where $S$ is the set of legal states, $s_{0}=\left(\left(\ell_{1}^{0}, \ell_{2}^{0}\right), v_{0}\right)$, where $v_{0}$ maps each clock to 0 , and $\rightarrow$ is defined by

Local action: $\left(\left(\ell_{1}, \ell_{2}\right), v\right) \stackrel{a}{\rightarrow}\left(\left(\ell_{1}^{\prime}, \ell_{2}\right), v^{\prime}\right)$ iff $a \in \Sigma_{1} \backslash \Sigma_{2}^{\ddagger}, \ell_{1} \stackrel{g, a, r}{\rightarrow} \ell_{1}^{\prime}, v \models g, v^{\prime}=v[r]$ and $v^{\prime} \models \operatorname{Inv} v_{1}\left(\ell_{1}^{\prime}\right)$, and similarly for a local action in $\Sigma_{2} \backslash \Sigma_{1}^{\nexists}$,

Synchronization: $\left(\left(\ell_{1}, \ell_{2}\right), v\right) \stackrel{a}{\rightarrow}\left(\left(\ell_{1}^{\prime}, \ell_{2}^{\prime}\right), v^{\prime}\right)$ iff $a \neq \varepsilon, \ell_{1} \stackrel{g_{1}, a, r_{1}}{\longrightarrow} \ell_{1}^{\prime}, \ell_{2} \stackrel{g_{2}, a, r_{2}}{\longrightarrow} \ell_{2}^{\prime}, v \models$ $g_{1} \wedge g_{2}, v^{\prime}=v\left[r_{1} \cup r_{2}\right]$ and $v^{\prime} \models \operatorname{Inv}_{1}\left(\ell_{1}^{\prime}\right) \wedge \operatorname{Inv}_{2}\left(\ell_{2}^{\prime}\right)$,

Time delay: $\forall d \in \mathbb{R}_{\geq 0},\left(\left(\ell_{1}, \ell_{2}\right), v\right) \stackrel{d}{\rightarrow}\left(\left(\ell_{1}, \ell_{2}\right), v+d\right)$ iff $\forall d^{\prime} \in[0, d], v+d^{\prime} \models \operatorname{Inv}_{1}\left(\ell_{1}\right) \wedge$ $\operatorname{Inv} v_{2}\left(\ell_{2}\right)$. 


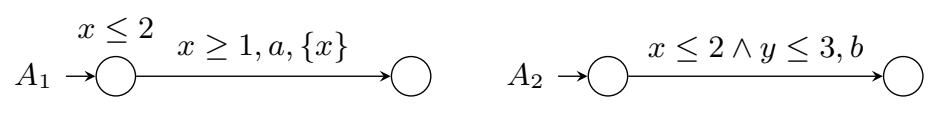

Figure 1: $A_{2}$ could avoid reading clock $x$ which belongs to $A_{1}$.

A run of an NTA is an initial path in its TTS. The semantics of a TA $A$ alone can also be given as a TTS denoted by $\operatorname{TTS}(A)$ with only local actions and delay. A TA is non-Zeno iff for every infinite timed word $w$ generated by a run, time diverges (i.e. $\delta(w)=\infty$ ). This is a common assumption for TA. In the sequel, we always assume that the TA we deal with are non-Zeno.

Remark 1.3. Let $A_{1} \| A_{2}$ be such that $X_{1} \cap X_{2}=\emptyset$. Then $\operatorname{TTS}\left(A_{1}\right) \otimes \operatorname{TTS}\left(A_{2}\right)$ is isomorphic to $\operatorname{TTS}\left(A_{1} \| A_{2}\right)$. This is not true in general when $X_{1} \cap X_{2} \neq \emptyset$. For example, in Fig. 2. taking $b$ at time 0.5 and $e$ at time 1 is possible in $\operatorname{TTS}\left(A_{1}\right) \otimes \operatorname{TTS}\left(A_{2}\right)$ but not in $\operatorname{TTS}\left(A_{1} \| A_{2}\right)$, since $b$ resets $x$ which is tested by $e$.

\section{NeEd For Shared Clocks}

2.1. Problem Setting. We are interested in detecting the cases where it is possible to avoid sharing clocks, so that the model can be implemented using no other synchronization than those explicitly described by common actions.

In this paper, we consider only the case of a network of two TA, $A_{1} \| A_{2}$, such that $A_{1}$ does not read the clocks reset by $A_{2}$, and $A_{2}$ may read the clocks reset by $A_{1}$. We want to know whether $A_{2}$ really needs to read these clocks, or if another NTA $A_{1}^{\prime} \| A_{2}^{\prime}$ could achieve the same behavior as $A_{1} \| A_{2}$ without using shared clocks.

A first remark is that our problem makes sense only if we insist on the distributed nature of the system, made of two separate components. On the other hand, if the composition operator is simply used as a convenient syntax for describing a system that is actually implemented on a single sequential component, then a simple product automaton would perfectly describe the system and every clock becomes local.

So, let us consider the example of Fig. 1, made of two TA, supposed to describe two separate components. Remark that $A_{2}$ reads clock $x$ which is reset by $A_{1}$. But a simple analysis shows that this reading could be avoided: because of the condition on its clock $y$, $A_{2}$ can only take transition $b$ before time 3 ; but $x$ cannot reach value 2 before time 3 , since it must be reset between time 1 and 2. Thus, forgetting the condition on $x$ in $A_{2}$ would not change the behavior of the system.

2.2. Transmitting Information during Synchronizations. Consider now the example of Fig. 2. Here also $A_{2}$ reads clock $x$ which is reset by $A_{1}$, and here also this reading could be avoided. The idea is that $A_{1}$ could transmit the value of $x$ when synchronizing, and afterwards any reading of $x$ in $A_{2}$ can be replaced by the reading of a new clock $x^{\prime}$ dedicated to storing the value of $x$ which is copied on the synchronization. Therefore $A_{2}$ can be replaced by $A_{2}^{\prime}$ pictured in Fig. 2 , while preserving the behavior of the NTA, but also the behavior of $A_{2}$ w.r.t. $A_{1}$.

We claim that we cannot avoid reading $x$ without this copy of clock. Indeed, after the synchronization, the maximal delay in the current location depends on the exact value of 

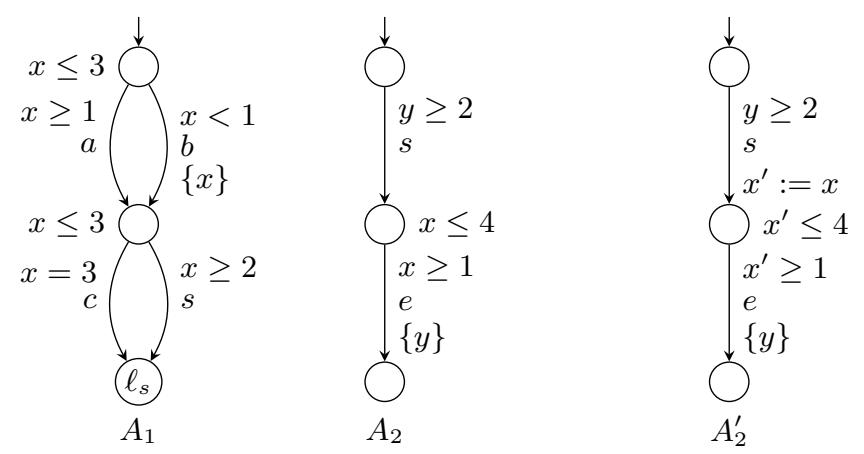

Figure 2: $A_{2}$ reads $x$ which belongs to $A_{1}$ and $A_{2}^{\prime}$ does not.

$x$, and even if we find a mechanism to allow $A_{2}^{\prime}$ to move to different locations according to the value of $x$ at synchronization time, infinitely many locations would be required (for example, if $s$ occurs at time $2, x$ may have any value in $(1,2])$.

Coding Transmission of Information. In order to model the transmission of information during synchronizations, we allow $A_{1}^{\prime}$ and $A_{2}^{\prime}$ to use a larger synchronization alphabet than $A_{1}$ and $A_{2}$. This allows $A_{1}^{\prime}$ to transmit discrete information, like its current location, to $A_{2}^{\prime}$.

But we saw that $A_{1}^{\prime}$ also needs to transmit the exact value of its clocks, which requires a more general mechanism than the simple clock resets. For this we allow an automaton to copy its neighbor's clocks into local clocks during synchronizations. This is denoted as updates of the form $x^{\prime}:=x$ in $A_{2}^{\prime}$ (see Fig. 2). This feature is a bit unusual but has already been studied: it is a restricted class of updatable timed automata as defined in [BDFP04]. Moreover, as shown in BDFP04, the class we consider, without comparisons of clocks and with only equalities in the updates is not more expressive than classical TA for the sequential semantics (any updatable TA of the class is bisimilar to a classical TA), and the emptiness problem is PSPACE-complete, as in the case of classical TAs.

Semantics. TTS $\left(A_{1} \| A_{2}\right)$ can be defined as previously, with the difference that the synchronizations are now defined by: $\left(\left(\ell_{1}, \ell_{2}\right), v\right) \stackrel{a}{\rightarrow}\left(\left(\ell_{1}^{\prime}, \ell_{2}^{\prime}\right), v^{\prime}\right)$ iff $\ell_{1} \stackrel{g_{1}, a, r_{1}}{\longrightarrow}{ }_{1} \ell_{1}^{\prime}, \ell_{2} \stackrel{g_{2}, a, r_{2}, u}{\longrightarrow}{ }_{2} \ell_{2}^{\prime}$ where $u$ is a partial function from $X_{2}$ to $X_{1}, v \models g_{1} \wedge g_{2}, v^{\prime}=\left(v\left[r_{1} \cup r_{2}\right]\right)[u]$, and $v^{\prime} \models$ $\operatorname{Inv}\left(\ell_{1}^{\prime}\right) \wedge \operatorname{Inv}\left(\ell_{2}^{\prime}\right)$. The valuation $v[u]$ is defined by $v[u](x)=v(u(x))$ if $u(x)$ is defined, and $v[u](x)=v(x)$ otherwise.

Here, we choose to apply the reset $r_{1} \cup r_{2}$ before the update $u$, because we are interested in sharing the state reached in $A_{1}$ after the synchronization, and $r_{1}$ may reset some clocks in $C_{1} \subseteq X_{1}$.

2.3. Towards a Formalization of the Problem. We want to know whether $A_{2}$ really needs to read the clocks reset by $A_{1}$, or if another NTA $A_{1}^{\prime} \| A_{2}^{\prime}$ could achieve the same behavior as $A_{1} \| A_{2}$ without using shared clocks. It remains to formalize what we mean by "having the same behavior" in this context.

First, we impose that the locality of actions is preserved, i.e. $A_{1}^{\prime}$ uses the same set of local actions as $A_{1}$, and similarly for $A_{2}^{\prime}$ and $A_{2}$. For the synchronizations, we have explained earlier why we allow $A_{1}^{\prime}$ and $A_{2}^{\prime}$ to use a larger synchronization alphabet than $A_{1}$ 


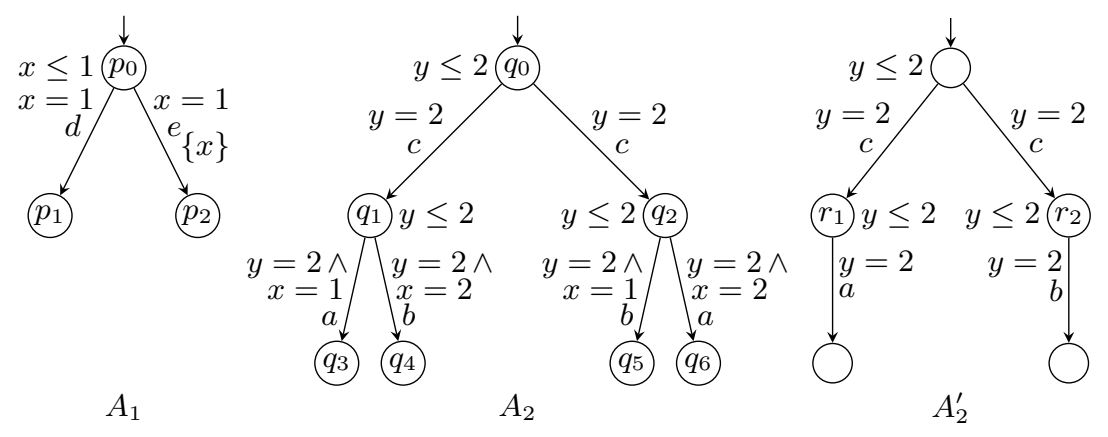

Figure 3: $A_{2}$ needs to read the clocks of $A_{1}$ and $\operatorname{TTS}\left(A_{1} \| A_{2}\right) \sim \operatorname{TTS}\left(A_{1} \| A_{2}^{\prime}\right)$.

and $A_{2}$. The correspondence between both alphabets will be done by a mapping $\psi$ (this point will be refined later).

Now we have to ensure that the behavior is preserved. The first idea that comes to mind is to impose bisimulation between $\psi\left(\operatorname{TTS}\left(A_{1}^{\prime} \| A_{2}^{\prime}\right)\right.$ ) (i.e. $\operatorname{TTS}\left(A_{1}^{\prime} \| A_{2}^{\prime}\right)$ with synchronization actions relabeled by $\psi$ ) and $\operatorname{TTS}\left(A_{1} \| A_{2}\right)$. But this is not sufficient, as illustrated by the example of Fig. 3 (where $\psi$ is the identity). Intuitively $A_{2}$ needs to read $x$ when in $q_{1}$ (and similarly in $q_{2}$ ) at time 2 , because this reading determines whether it will perform $a$ or $b$, and the value of $x$ cannot be inferred from its local state given by $q_{1}$ and the value of $y$. Anyway $\operatorname{TTS}\left(A_{1} \| A_{2}^{\prime}\right)$ is bisimilar to $\operatorname{TTS}\left(A_{1} \| A_{2}\right)$, and $A_{2}^{\prime}$ does not read $x$. For the bisimulation relation $\mathcal{R}$, it is sufficient to impose $\left(p_{1}, q_{1}\right) \mathcal{R}\left(p_{1}, r_{1}\right)$ and $\left(p_{2}, q_{1}\right) \mathcal{R}\left(p_{2}, r_{2}\right)$.

What we see here is that, from the point of view of $A_{2}$ and $A_{2}^{\prime}$, these two automata do not behave the same. As a matter of fact, when $A_{2}$ fires one edge labeled by $c$, it has not read $x$ yet, and there is still a possibility to fire $a$ or $b$, whereas when $A_{2}^{\prime}$ fires one edge labeled by $c$, there is no more choice afterwards. Therefore we need a relation between $A_{2}^{\prime}$ and $A_{2}$, and in the general case, a relation between $A_{1}^{\prime}$ and $A_{1}$ also.

\section{Contextual Timed Transition Systems}

As we are interested in representing a partial view of one of the components, we need to introduce another notion, that we call contextual timed transition system. This resembles the powerset construction used in game theory to capture the knowledge of an agent about another agent [Rei84].

Notations. $\mathbb{S}=\Sigma_{1}^{\ddagger} \cap \Sigma_{2}^{\ddagger}$ denotes the set of common actions. $Q_{1}$ denotes the set of states of $\operatorname{TTS}\left(A_{1}\right)$. When $s=\left(\left(\ell_{1}, \ell_{2}\right), v\right)$ is a state of $\operatorname{TTS}\left(A_{1} \| A_{2}\right)$, we also write $s=\left(s_{1}, s_{2}\right)$, where $s_{1}=\left(\ell_{1}, v_{\mid X_{1}}\right)$ is in $Q_{1}$, and $s_{2}=\left(\ell_{2}, v_{\mid X_{2} \backslash X_{1}}\right)$, where $v_{\mid X}$ is $v$ restricted to $X$.

Definition 3.1 $(\operatorname{UR}(s))$. Let $\operatorname{TTS}\left(A_{1}\right)=\left(Q_{1}, s_{0}, \Sigma_{1}, \rightarrow_{1}\right)$ and $s \in Q_{1}$. The set of states of $A_{1}$ reachable from $s$ by local actions in 0 delay (and therefore not observable by $A_{2}$ ) is denoted by $\mathrm{UR}(s)$ (for Unobservably Reachable) and defined as

$$
U R(s)=\left\{s^{\prime} \in Q_{1} \mid \exists w \in \mathrm{TW}_{0}\left(\Sigma_{1} \backslash \Sigma_{2}^{q}\right): s \stackrel{w}{\rightarrow}_{1} s^{\prime}\right\} .
$$




\subsection{Contextual TTS.}

Contextual States. The states of this contextual TTS are called contextual states. They can be regarded as possibly infinite sets of states of $\operatorname{TTS}\left(A_{1} \| A_{2}\right)$ for which $A_{2}$ is in the same location and has the same valuation over $X_{2} \backslash X_{1} . A_{2}$ may not be able to distinguish between some states $\left(s_{1}, s_{2}\right)$ and $\left(s_{1}^{\prime}, s_{2}\right)$. In $\operatorname{TTS}_{A_{1}}\left(A_{2}\right)$, these states are grouped into the same contextual state. However, when $X_{2} \cap X_{1} \neq \emptyset$, it may happen that $A_{2}$ is able to perform a local action or delay from $\left(s_{1}, s_{2}\right)$ and not from $\left(s_{1}^{\prime}, s_{2}\right)$, even if these states are grouped in a same contextual state.

Definition 3.2 (Contextual TTS). Let $\operatorname{TTS}\left(A_{1} \| A_{2}\right)=\left(Q, q_{0}, \Sigma_{1} \cup \Sigma_{2}, \Rightarrow\right)$. Then, the TTS of $A_{2}$ in the context of $A_{1}$, denoted by $\operatorname{TTS}_{A_{1}}\left(A_{2}\right)$, is the TTS $\left(S, s_{0},\left(\Sigma_{2} \backslash \mathbb{S}\right) \cup(\mathbb{S} \times\right.$ $\left.\left.Q_{1}\right), \rightarrow\right)$, where

- $S=\left\{\left(S_{1}, s_{2}\right) \mid \forall s_{1} \in S_{1},\left(s_{1}, s_{2}\right) \in Q\right\}$,

- $s_{0}=\left(S_{1}^{0}, s_{2}^{0}\right)$, s.t. $\left(s_{1}^{0}, s_{2}^{0}\right)=q_{0}$ and $S_{1}^{0}=\mathrm{UR}\left(s_{1}^{0}\right)$,

- $\rightarrow$ is defined by

- Local action: for any $a \in \Sigma_{2} \backslash \mathbb{S},\left(S_{1}, s_{2}\right) \stackrel{a}{\rightarrow}\left(S_{1}^{\prime}, s_{2}^{\prime}\right)$ iff $\exists s_{1} \in S_{1}:\left(s_{1}, s_{2}\right) \stackrel{a}{\Rightarrow}\left(s_{1}, s_{2}^{\prime}\right)$, and $S_{1}^{\prime}=\left\{s_{1} \in S_{1} \mid\left(s_{1}, s_{2}\right) \stackrel{a}{\Rightarrow}\left(s_{1}, s_{2}^{\prime}\right)\right\}$

- Synchronization: for any $\left(a, s_{1}^{\prime}\right) \in \mathbb{S} \times Q_{1},\left(S_{1}, s_{2}\right) \stackrel{a, s_{1}^{\prime}}{\longrightarrow}\left(\operatorname{UR}\left(s_{1}^{\prime}\right), s_{2}^{\prime}\right)$ iff $\exists s_{1} \in S_{1}$ : $\left(s_{1}, s_{2}\right) \stackrel{a}{\Rightarrow}\left(s_{1}^{\prime}, s_{2}^{\prime}\right)$

- Local delay: for any $d \in \mathbb{R}_{\geq 0},\left(S_{1}, s_{2}\right) \stackrel{d}{\rightarrow}\left(S_{1}^{\prime}, s_{2}^{\prime}\right)$ iff $\exists s_{1} \in S_{1}, \rho \in \operatorname{Paths}\left(\Sigma_{1} \backslash \Sigma_{2}^{q}, d\right)$ : $\left(s_{1}, s_{2}\right) \stackrel{\rho}{\Rightarrow}\left(s_{1}^{\prime}, s_{2}^{\prime}\right)$, and $S_{1}^{\prime}=\left\{s_{1}^{\prime} \mid \exists s_{1} \in S_{1}, \rho \in \operatorname{Paths}\left(\Sigma_{1} \backslash \Sigma_{2}^{\prime}, d\right):\left(s_{1}, s_{2}\right) \stackrel{\rho}{\Rightarrow}\left(s_{1}^{\prime}, s_{2}^{\prime}\right)\right\}$

For example, consider $A_{1}$ and $A_{2}$ of Fig. 3. The initial state is $\left(\left\{\left(p_{0}, 0\right)\right\},\left(q_{0}, 0\right)\right)$. From this contextual state, it is possible to delay 2 time units and reach the contextual state $\left(\left\{\left(p_{1}, 2\right),\left(p_{2}, 1\right)\right\},\left(q_{0}, 2\right)\right)$. Indeed, during this delay, $A_{1}$ has to perform either $e$ and reset $x$, or $d$. Now, from this contextual state, we can take an edge labeled by $c$, and reach $\left(\left\{\left(p_{1}, 2\right),\left(p_{2}, 1\right)\right\},\left(q_{1}, 2\right)\right)$. Lastly, from this new state, $a$ can be fired, because it is enabled by $\left(\left(p_{2}, 1\right),\left(q_{1}, 2\right)\right)$ in the TTS of the NTA, and the reached contextual state is $\left(\left\{\left(p_{2}, 1\right)\right\},\left(q_{3}, 2\right)\right)$.

Unrestricted Contextual TTS. We say that there is no restriction in $\operatorname{TTS}_{A_{1}}\left(A_{2}\right)$ if whenever a local step is possible from a reachable contextual state, then it is possible from all the states $\left(s_{1}, s_{2}\right)$ that are grouped into this contextual state. In the example above, there is a restriction in $\operatorname{TTS}_{A_{1}}\left(A_{2}\right)$ because we have seen that $a$ is enabled only by $\left(\left(p_{2}, 1\right),\left(q_{1}, 2\right)\right)$, and not by all states merged in $\left(\left\{\left(p_{1}, 2\right),\left(p_{2}, 1\right)\right\},\left(q_{1}, 2\right)\right)$. Formally, we use the predicate noRestriction $_{A_{1}}\left(A_{2}\right)$ defined as follows.

Definition 3.3 (noRestriction $_{A_{1}}\left(A_{2}\right)$ ). The predicate noRestriction $A_{1}\left(A_{2}\right)$ holds iff for any reachable state $\left(S_{1}, s_{2}\right)$ of $\operatorname{TTS}_{A_{1}}\left(A_{2}\right)$, both

- $\forall a \in \Sigma_{2} \backslash \mathbb{S},\left(S_{1}, s_{2}\right) \stackrel{a}{\rightarrow}\left(S_{1}^{\prime}, s_{2}^{\prime}\right) \Longleftrightarrow \forall s_{1} \in S_{1},\left(s_{1}, s_{2}\right) \stackrel{a}{\Rightarrow}\left(s_{1}, s_{2}^{\prime}\right)$, and

- $\forall d \in \mathbb{R}_{\geq 0},\left(S_{1}, s_{2}\right) \stackrel{d}{\rightarrow} \Longleftrightarrow \forall s_{1} \in S_{1}, \exists \rho \in \operatorname{Paths}\left(\Sigma_{1} \backslash \Sigma_{2}^{g}, d\right):\left(s_{1}, s_{2}\right) \stackrel{\rho}{\Rightarrow}$

Remark 3.4. If $A_{2}$ does not read $X_{1}$, then there is no restriction in $\operatorname{TTS}_{A_{1}}\left(A_{2}\right)$. 


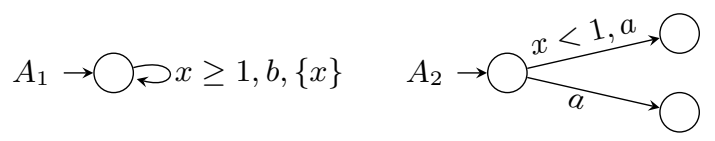

Figure 4: $\operatorname{TTS}_{Q_{1}}\left(A_{1}\right) \otimes \operatorname{TTS}_{A_{1}}\left(A_{2}\right) \approx \operatorname{TTS}_{Q_{1}}\left(A_{1} \| A_{2}\right)$, although there is a restriction in $\operatorname{TTS}_{A_{1}}\left(A_{2}\right)$

Sharing of Information During Synchronizations. Later we assume that during a synchronization, $A_{1}$ is allowed to transmit all its state to $A_{2}$, that is why, in $\operatorname{TTS}_{A_{1}}\left(A_{2}\right)$, we distinguish the states reached after a synchronization according to the state reached in $A_{1}$. We also label the synchronization edges by a pair $\left(a, s_{1}\right) \in \mathbb{S} \times Q_{1}$ where $a$ is the action and $s_{1}$ the state reached in $A_{1}$.

For the sequel, let $\operatorname{TTS}_{Q_{1}}\left(A_{1}\right)\left(\right.$ resp. $\left.\operatorname{TTS}_{Q_{1}}\left(A_{1} \| A_{2}\right)\right)$ denote $\operatorname{TTS}\left(A_{1}\right)\left(\right.$ resp. $\operatorname{TTS}\left(A_{1} \|\right.$ $\left.A_{2}\right)$ ) where the synchronization edges are labeled by $\left(a, s_{1}\right)$, where $a \in \mathbb{S}$ is the action, and $s_{1}$ is the state reached in $A_{1}$.

We can now state a nice property of unrestricted contextual TTS that is similar to the distributivity of TTS over the composition when considering TA with disjoint sets of clocks (see Remark 1.3). We say that a TA is deterministic if it has no $\varepsilon$-transition and for any location $\ell$ and action $a$, there is at most one edge labeled by $a$ from $\ell$.

Lemma 3.5. If there is no restriction in $\operatorname{TTS}_{A_{1}}\left(A_{2}\right)$, then $\operatorname{TTS}_{Q_{1}}\left(A_{1}\right) \otimes \operatorname{TTS}_{A_{1}}\left(A_{2}\right) \approx$ $\mathrm{TTS}_{Q_{1}}\left(A_{1} \| A_{2}\right)$. Moreover, when $A_{2}$ is deterministic, this condition becomes necessary.

The example of Fig. 4 shows that the reciprocal does not hold when $A_{2}$ is not deterministic. In order to prove Lemma 3.5, we first present two propositions. The first one relates the reachable states of $\operatorname{TTS}_{A_{1}}\left(A_{2}\right)$ with those of $\operatorname{TTS}_{Q_{1}}\left(A_{1}\right) \otimes \operatorname{TTS}_{A_{1}}\left(A_{2}\right)$.

\section{Proposition 3.6.}

(1) For any reachable state $\left(S_{1}, s_{2}\right)$ of $\operatorname{TTS}_{A_{1}}\left(A_{2}\right)$, $s_{1} \in S_{1} \Longrightarrow\left(s_{1},\left(S_{1}, s_{2}\right)\right)$ is a reachable state of $\operatorname{TTS}_{Q_{1}}\left(A_{1}\right) \otimes \operatorname{TTS}_{A_{1}}\left(A_{2}\right)$

(2) noRestriction $_{A_{1}}\left(A_{2}\right)$ iff for any reachable state $\left(S_{1}, s_{2}\right)$ of $\operatorname{TTS}_{A_{1}}\left(A_{2}\right)$, $s_{1} \in S_{1} \Longleftrightarrow\left(s_{1},\left(S_{1}, s_{2}\right)\right)$ is a reachable state of $\operatorname{TTS}_{Q_{1}}\left(A_{1}\right) \otimes \operatorname{TTS}_{A_{1}}\left(A_{2}\right)$

Proof. (1) For any reachable state $\left(S_{1}, s_{2}\right)$, let us denote by $P\left(S_{1}, s_{2}\right)$ the fact that for any $s_{1} \in S_{1},\left(s_{1},\left(S_{1}, s_{2}\right)\right)$ is reachable in $\operatorname{TTS}_{Q_{1}}\left(A_{1}\right) \otimes \operatorname{TTS}_{A_{1}}\left(A_{2}\right)$. We give a recursive proof. First, the initial state $\left(S_{1}^{0}, s_{2}^{0}\right)$ satisfies $P\left(S_{1}^{0}, s_{2}^{0}\right)$ because for any $s_{1} \in S_{1}^{0}=\operatorname{UR}\left(s_{1}^{0}\right)$, $\exists w \in \mathrm{TW}_{0}\left(\Sigma_{1} \backslash \Sigma_{2}^{q}\right): s_{1}^{0} \stackrel{w}{\rightarrow}_{1} s_{1}$ and hence $\left(s_{1}^{0},\left(S_{1}^{0}, s_{2}^{0}\right)\right) \stackrel{w}{\rightarrow}\left(s_{1},\left(S_{1}^{0}, s_{2}^{0}\right)\right)$. Then, assume some reachable state $\left(S_{1}, s_{2}\right)$ of $\operatorname{TTS}_{A_{1}}\left(A_{2}\right)$ satisfies $P\left(S_{1}, s_{2}\right)$ and show that any state $\left(S_{1}^{\prime}, s_{2}^{\prime}\right)$ reachable in one step from $\left(S_{1}, s_{2}\right)$ also satisfies $P\left(S_{1}^{\prime}, s_{2}^{\prime}\right)$. There can be three kinds of steps from $\left(S_{1}, s_{2}\right)$ in $\operatorname{TTS}_{A_{1}}\left(A_{2}\right)$.

(1) If for some $a \in \Sigma_{2} \backslash \mathbb{S},\left(S_{1}, s_{2}\right) \stackrel{a}{\rightarrow}\left(S_{1}^{\prime}, s_{2}^{\prime}\right)$, then for any $s_{1}^{\prime} \in S_{1}^{\prime} \subseteq S_{1},\left(s_{1}^{\prime},\left(S_{1}, s_{2}\right)\right) \stackrel{a}{\rightarrow}$ $\left(s_{1}^{\prime},\left(S_{1}^{\prime}, s_{2}^{\prime}\right)\right)$, i.e. $P\left(S_{1}^{\prime}, s_{2}^{\prime}\right)$ holds.

(2) If for some $\left(a, s_{1}^{\prime}\right) \in \mathbb{S} \times Q_{1},\left(S_{1}, s_{2}\right) \stackrel{a, s_{1}^{\prime}}{\longrightarrow}\left(S_{1}^{\prime}, s_{2}^{\prime}\right)$, then $S_{1}^{\prime}=\mathrm{UR}\left(s_{1}^{\prime}\right)$, and for some $s_{1} \in S_{1},\left(s_{1},\left(S_{1}, s_{2}\right)\right) \stackrel{a, s_{1}^{\prime}}{\longrightarrow}\left(s_{1}^{\prime},\left(S_{1}^{\prime}, s_{2}^{\prime}\right)\right)$. By the same reasoning as for $\left(S_{1}^{0}, s_{2}^{0}\right)$, for any $s_{1}^{\prime \prime} \in S_{1}^{\prime}=\mathrm{UR}\left(s_{1}^{\prime}\right), \exists w \in \mathrm{TW}_{0}\left(\Sigma_{1} \backslash \Sigma_{2}^{q}\right):\left(s_{1}^{\prime},\left(S_{1}^{\prime}, s_{2}^{\prime}\right)\right) \stackrel{w}{\rightarrow}\left(s_{1}^{\prime \prime},\left(S_{1}^{\prime}, s_{2}^{\prime}\right)\right)$. Hence $P\left(S_{1}^{\prime}, s_{2}^{\prime}\right)$ holds. 
(3) If for some $d \in \mathbb{R}_{\geq 0},\left(S_{1}, s_{2}\right) \stackrel{d}{\rightarrow}\left(S_{1}^{\prime}, s_{2}^{\prime}\right)$, then $\exists d_{1} \leq d:\left(S_{1}, s_{2}\right) \stackrel{d_{1}}{\rightarrow}\left(S_{1}^{1}, s_{2}^{1}\right) \wedge \exists s_{1}^{1} \in$ $S_{1}^{1}, s_{1} \in S_{1}:\left(s_{1}, s_{2}\right) \stackrel{d_{1}}{\Rightarrow}\left(s_{1}^{1}, s_{2}^{1}\right)$, that is $\left(s_{1}^{1},\left(S_{1}^{1}, s_{2}^{1}\right)\right)$ is reachable, and by timedeterminism, $\left(S_{1}^{1}, s_{2}^{1}\right) \stackrel{d-d_{1}}{\longrightarrow}\left(S_{1}^{\prime}, s_{2}^{\prime}\right)$.

For the third case, take $d_{1}$ small enough (but strictly positive) so that $S_{1}^{1}=\left\{s_{1}^{\prime} \mid \exists s_{1} \in\right.$ $\left.S_{1}:\left(s_{1}, s_{2}\right) \stackrel{d_{1}}{\Rightarrow}\left(s_{1}^{1}, s_{2}^{1}\right) \wedge s_{1}^{\prime} \in \operatorname{UR}\left(s_{1}^{1}\right)\right\}$. That is, after some local actions that take no time, $A_{1}$ is able to perform a delay $d_{1}$ during which no local action is enabled (such $d_{1}$ exists because of the non-zenoness assumption). With such $d_{1}$, any state $s_{1}^{\prime} \in S_{1}^{1}$ is such that $s_{1}^{\prime} \in \operatorname{UR}\left(s_{1}^{1}\right)$ for some $s_{1}^{1}$ so that $\left(s_{1}^{1},\left(S_{1}^{1}, s_{2}^{1}\right)\right)$ is reachable. Therefore, $\exists w \in \mathrm{TW}_{0}\left(\Sigma_{1} \backslash \Sigma_{2}^{q}\right)$ : $\left(s_{1}^{1},\left(S_{1}^{1}, s_{2}^{1}\right)\right) \stackrel{w}{\rightarrow}\left(s_{1}^{\prime},\left(S_{1}^{1}, s_{2}^{1}\right)\right)$ and hence $P\left(S_{1}^{1}, s_{2}^{1}\right)$ holds.

Since $A_{1}$ is not Zeno, any delay in $\operatorname{TTS}_{A_{1}}\left(A_{2}\right)$ can be cut into a finite number of such smaller global delays. Hence, for any $\left(S_{1}, s_{2}\right)$ that satisfies $P\left(S_{1}, s_{2}\right)$, for any $d \in \mathbb{R}_{\geq 0}$ such that $\left(S_{1}, s_{2}\right) \stackrel{d}{\rightarrow}\left(S_{1}^{\prime}, s_{2}^{\prime}\right), P\left(S_{1}^{\prime}, s_{2}^{\prime}\right)$ holds.

$(2, \Rightarrow)(1)$ already gives that $\forall s_{1} \in S_{1},\left(s_{1},\left(S_{1}, s_{2}\right)\right)$ is a reachable state. So it remains to prove that, when noRestriction $A_{1}\left(A_{2}\right)$, if $\left(s_{1},\left(S_{1}, s_{2}\right)\right)$ is a reachable state, then $s_{1} \in S_{1}$. We say that a reachable state $s=\left(s_{1},\left(S_{1}, s_{2}\right)\right)$ satisfies $P(s)$ iff $s_{1} \in S_{1}$.

Assume noRestriction $A_{1}\left(A_{2}\right)$ and $s=\left(s_{1},\left(S_{1}, s_{2}\right)\right)$ is a reachable state that satisfies $P(s)$. Then, any state $s^{\prime}$ reachable in one step from $s$ by some local action or delay $a \in$ $\left(\Sigma_{1} \cup \Sigma_{2}\right) \backslash \mathbb{S} \cup \mathbb{R}_{\geq 0}$ or by some synchronization $\left(a, s_{1}^{\prime}\right) \in \mathbb{S} \times Q_{1}$ matches one of the following cases:

- if $a \in \Sigma_{1} \backslash \Sigma_{2}^{q}$, then $s^{\prime}=\left(s_{1}^{\prime},\left(S_{1}, s_{2}\right)\right)$ such that $s_{1}^{\prime} \in \operatorname{UR}\left(s_{1}\right) \subseteq S_{1}$ (by construction, $\left.s_{1} \in S_{1} \Longrightarrow \mathrm{UR}\left(s_{1}\right) \subseteq S_{1}\right)$,

- if $a \in \Sigma_{2} \backslash \Sigma_{1}$, then $s^{\prime}=\left(s_{1},\left(S_{1}, s_{2}^{\prime}\right)\right)$,

- if $a \in \mathbb{R}_{\geq 0}$, then $s^{\prime}=\left(s_{1}^{\prime},\left(S_{1}^{\prime}, s_{2}^{\prime}\right)\right)$, where $s_{1}^{\prime}$ such that $\left(s_{1}, s_{2}\right) \stackrel{a}{\Rightarrow}\left(s_{1}^{\prime}, s_{2}^{\prime}\right)$ is in $S_{1}^{\prime}=\left\{q_{1}^{\prime} \mid\right.$ $\left.\exists q_{1} \in S_{1}, \rho \in \operatorname{Paths}\left(\Sigma_{1} \backslash \Sigma_{2}^{q}, a\right):\left(q_{1}, s_{2}\right) \stackrel{\rho}{\Rightarrow}\left(q_{1}^{\prime}, s_{2}^{\prime}\right)\right\}$,

- if $\left(a, s_{1}^{\prime}\right) \in\left(\mathbb{S} \times Q_{1}\right)$, then $s^{\prime}=\left(s_{1}^{\prime},\left(\operatorname{UR}\left(s_{1}^{\prime}\right), s_{2}^{\prime}\right)\right)$.

Therefore, any state $s^{\prime}$ reached in one step from $s$ also satisfies $P\left(s^{\prime}\right)$, and recursively, since the initial state $s_{0}=\left(s_{1}^{0},\left(\mathrm{UR}\left(s_{1}^{0}\right), s_{2}^{0}\right)\right)$ satisfies $P\left(s_{0}\right)$, any reachable state $s$ of $\operatorname{TTS}_{Q_{1}}\left(A_{1}\right) \otimes$ $\operatorname{TTS}_{A_{1}}\left(A_{2}\right)$ satisfies $P(s)$.

$(2, \Leftarrow)$ By contradiction, assume there is a restriction in state $\left(S_{1}, s_{2}\right)$ for local delay or action $a \in\left(\Sigma_{2} \backslash \Sigma_{1}\right) \cup \mathbb{R}_{\geq 0}$ i.e. $a$ is possible from some state $\left(s_{1}^{\prime}, s_{2}\right)$ but not from another state $\left(s_{1}, s_{2}\right)$ such that $s_{1}^{\prime}, s_{1} \in S_{1}$. Then, after performing a from $\left(s_{1},\left(S_{1}, s_{2}\right)\right)$, that is reachable according to Proposition [3.6, we reach state $\left(s_{1},\left(S_{1}^{\prime}, s_{2}^{\prime}\right)\right)$ such that $s_{1} \notin S_{1}^{\prime}$.

Proposition 3.7. If noRestriction $A_{A_{1}}\left(A_{2}\right)$ then, for any timed word $w$ over $\left(\Sigma_{2} \backslash \mathbb{S}\right) \cup\left(\mathbb{S} \times Q_{1}\right)$, there exists at most one $S_{1}$ such that, for some $s_{2},\left(S_{1}^{0}, s_{2}^{0}\right) \stackrel{w}{\rightarrow}\left(S_{1}, s_{2}\right)$ in $\operatorname{TTS}_{A_{1}}\left(A_{2}\right)$ (i.e. $S_{1}$ is uniquely determined by $w$, whatever the structure of $\left.A_{2}\right)$.

Proof. Assuming noRestriction $A_{1}\left(A_{2}\right)$, we show that, for any $\left(S_{1}^{1}, s_{2}^{1}\right)$ reachable in $\operatorname{TTS}_{A_{1}}\left(A_{2}\right)$, for any action or delay in $\left(\Sigma_{2} \backslash \mathbb{S}\right) \cup\left(\mathbb{S} \times Q_{1}\right) \cup \mathbb{R}_{\geq 0}$, there is at most one $S_{1}$ such that, for some $s_{2},\left(S_{1}, s_{2}\right)$ is a successor of $\left(S_{1}^{1}, s_{2}^{1}\right)$ by this action.

Indeed, by construction, and since there is no restriction,

- any successor of $\left(S_{1}^{1}, s_{2}^{1}\right)$ by a local action is of the form $\left(S_{1}^{1}, s_{2}^{\prime}\right)$,

- any successor of $\left(S_{1}^{1}, s_{2}^{1}\right)$ by a synchronization $\left(a, s_{1}^{\prime}\right)$ is of the form $\left(\operatorname{UR}\left(s_{1}^{\prime}\right), s_{2}^{\prime}\right)$,

- any successor of $\left(S_{1}^{1}, s_{2}^{1}\right)$ by a delay $d$ is of the form $\left(S_{1}, s_{2}^{\prime}\right)$ with $S_{1}=\left\{s_{1}^{\prime} \mid \exists \rho \in\right.$ $\left.\operatorname{Paths}\left(\Sigma_{1} \backslash \Sigma_{2}^{\ddagger}, d\right), s_{1} \in S_{1}^{1}: s_{1} \stackrel{\rho}{\rightarrow}_{1} s_{1}^{\prime}\right\}$. 
Therefore, for any possible action or delay, $S_{1}$ does not depend on the state of $A_{2}$, and is uniquely determined by this action or delay.

Since $\left(S_{1}^{0}, s_{2}^{0}\right)$ is unique, for any timed word $w$ over $\left(\Sigma_{2} \backslash \mathbb{S}\right) \cup\left(\mathbb{S} \times Q_{1}\right)$, either $w$ does not describe a valid path in $\operatorname{TTS}_{A_{1}}\left(A_{2}\right)$, or there exists a unique $S_{1}$ such that for some $s_{2}$, $\left(S_{1}^{0}, s_{2}^{0}\right) \stackrel{w}{\rightarrow}\left(S_{1}, s_{2}\right)$ in $\operatorname{TTS}_{A_{1}}\left(A_{2}\right)$.

We can now prove Lemma 3.5 .

Proof of Lemma 3.5. Assume noRestriction $A_{1}\left(A_{2}\right)$, and define relation $\mathcal{R}$ as $\left(s_{1},\left(S_{1}, s_{2}\right)\right) \mathcal{R}$ $\left(s_{1}^{\prime}, s_{2}^{\prime}\right) \stackrel{\text { def }}{\Longleftrightarrow} s_{1}=s_{1}^{\prime} \wedge s_{2}=s_{2}^{\prime}$, for any reachable states $\left(s_{1},\left(S_{1}, s_{2}\right)\right)$ of $\operatorname{TTS}_{Q_{1}}\left(A_{1}\right) \otimes$ $\operatorname{TTS}_{A_{1}}\left(A_{2}\right)$ and $\left(s_{1}^{\prime}, s_{2}^{\prime}\right)$ of $\operatorname{TTS}_{Q_{1}}\left(A_{1} \| A_{2}\right)$. By Proposition [3.6. since $\left(s_{1},\left(S_{1}, s_{2}\right)\right)$ is reachable, $s_{1} \in S_{1}$. We show that $\mathcal{R}$ is a strong timed bisimulation.

First, the initial states are $\mathcal{R}$-related: $\left(s_{1}^{0},\left(S_{1}^{0}, s_{2}^{0}\right)\right) \mathcal{R}\left(s_{1}^{0}, s_{2}^{0}\right)$. Then, if $\left(s_{1},\left(S_{1}, s_{2}\right)\right) \mathcal{R}$ $\left(s_{1}^{\prime}, s_{2}^{\prime}\right)$, four kinds of steps are possible:

- if for some $a \in \Sigma_{1} \backslash \Sigma_{2}^{q},\left(s_{1},\left(S_{1}, s_{2}\right)\right) \stackrel{a}{\rightarrow}\left(s_{1}^{\prime},\left(S_{1}, s_{2}\right)\right)$, then $\left(s_{1}, s_{2}\right) \stackrel{a}{\Rightarrow}\left(s_{1}^{\prime}, s_{2}\right)$ and $\left(s_{1}^{\prime},\left(S_{1}, s_{2}\right)\right) \mathcal{R}\left(s_{1}^{\prime}, s_{2}\right)$, and conversely.

- if for some $a \in \Sigma_{2} \backslash \Sigma_{1},\left(s_{1},\left(S_{1}, s_{2}\right)\right) \stackrel{a}{\rightarrow}\left(s_{1},\left(S_{1}, s_{2}^{\prime}\right)\right)$, then, $\forall s_{11} \in S_{1},\left(s_{11}, s_{2}\right) \stackrel{a}{\Rightarrow}\left(s_{11}, s_{2}^{\prime}\right)$ (because noRestriction $\left._{A_{1}}\left(A_{2}\right)\right)$, and in particular, $\left(s_{1}, s_{2}\right) \stackrel{a}{\Rightarrow}\left(s_{1}, s_{2}^{\prime}\right)$ and $\left(s_{1},\left(S_{1}, s_{2}^{\prime}\right)\right) \mathcal{R}$ $\left(s_{1}, s_{2}^{\prime}\right)$, and conversely.

- if for some $\left(a, s_{1}^{\prime}\right) \in \mathbb{S} \times Q_{1},\left(s_{1},\left(S_{1}, s_{2}\right)\right) \stackrel{a, s_{1}^{\prime}}{\longrightarrow}\left(s_{1}^{\prime},\left(S_{1}^{\prime}, s_{2}^{\prime}\right)\right)$, then $\left(s_{1}, s_{2}\right) \stackrel{a, s_{1}^{\prime}}{\Longrightarrow}\left(s_{1}^{\prime}, s_{2}^{\prime}\right)$ and $\left(s_{1}^{\prime},\left(S_{1}^{\prime}, s_{2}^{\prime}\right)\right) \mathcal{R}\left(s_{1}^{\prime}, s_{2}^{\prime}\right)$, and conversely.

- if for some $d \in \mathbb{R}_{\geq 0},\left(s_{1},\left(S_{1}, s_{2}\right)\right) \stackrel{d}{\rightarrow}\left(s_{1}^{\prime},\left(S_{1}^{\prime}, s_{2}^{\prime}\right)\right)$, then $\left(s_{1}, s_{2}\right) \stackrel{d}{\Rightarrow}\left(s_{1}^{\prime}, s_{2}^{\prime}\right)$ (because noRestriction $\left._{A_{1}}\left(A_{2}\right)\right)$, and $\left(s_{1}^{\prime},\left(S_{1}^{\prime}, s_{2}^{\prime}\right)\right) \mathcal{R}\left(s_{1}^{\prime}, s_{2}^{\prime}\right)$, and conversely.

Now assume $A_{2}$ is deterministic. Let relation $\mathcal{R}$ be a strong timed bisimulation between $\operatorname{TTS}_{Q_{1}}\left(A_{1}\right) \otimes \operatorname{TTS}_{A_{1}}\left(A_{2}\right)$ and $\operatorname{TTS}_{Q_{1}}\left(A_{1} \| A_{2}\right)$.

By contradiction, assume there is a restriction in $\operatorname{TTS}_{A_{1}}\left(A_{2}\right)$. Then there is a reachable state $\left(S_{1}, s_{2}\right)$ of $\operatorname{TTS}_{A_{1}}\left(A_{2}\right)$, and a local delay or action $a \in\left(\Sigma_{2} \backslash \Sigma_{1}\right) \cup \mathbb{R}_{\geq 0}$ such that, for some $s_{1}, s_{1}^{\prime} \in S_{1},\left(s_{1}, s_{2}\right)$ enables $a$ in $\operatorname{TTS}_{Q_{1}}\left(A_{1} \| A_{2}\right)$, whereas $\left(s_{1}^{\prime}, s_{2}\right)$ does not.

By definition of a bisimulation, there also exist two states $\left(p_{1},\left(P_{1}, p_{2}\right)\right)$ and $\left(p_{1}^{\prime},\left(P_{1}^{\prime}, p_{2}^{\prime}\right)\right.$ such that $\left(p_{1},\left(P_{1}, p_{2}\right)\right) \mathcal{R}\left(s_{1}, s_{2}\right)$ and $\left(p_{1}^{\prime},\left(P_{1}^{\prime}, p_{2}^{\prime}\right)\right) \mathcal{R}\left(s_{1}^{\prime}, s_{2}\right)$. That is, in particular, $\left(p_{1}^{\prime},\left(P_{1}^{\prime}, p_{2}^{\prime}\right)\right)$ does not enable $a$. Moreover, these states can be chosen so that they are reached by the same timed word over $\left(\Sigma_{2} \backslash \mathbb{S}\right) \cup\left(\mathbb{S} \times Q_{1}\right)$, and since $A_{2}$ is deterministic, $p_{2}=p_{2}^{\prime}=s_{2}$.

Now, we can assume that $\left(S_{1}, s_{2}\right)$ is chosen so that it is the first state with a restriction along an initial path. Then, the paths to $\left(P_{1}, s_{2}\right)$ and $\left(P_{1}^{\prime}, s_{2}\right)$ generate the same timed word over $\left(\Sigma_{2} \backslash \mathbb{S}\right) \cup\left(\mathbb{S} \times Q_{1}\right)$, and by Proposition 3.7, $P_{1}=P_{1}^{\prime}=S_{1}$.

Therefore, we have shown the existence of a state $\left(p_{1}^{\prime},\left(S_{1}, s_{2}\right)\right)$ in $\operatorname{TTS}_{Q_{1}}\left(A_{1}\right) \otimes \operatorname{TTS}_{A_{1}}\left(A_{2}\right)$ that does not enable $a$, which means that $\left(S_{1}, s_{2}\right)$ does not enable $a$ in $\operatorname{TTS}_{A_{1}}\left(A_{2}\right)$. This contradicts the fact that there exists $s_{1} \in S_{1}$ such that $\left(s_{1}, s_{2}\right)$ enables $a$.

We are now in condition to formalize our problem.

3.2. Need for Shared Clocks Revisited. We have argued in Section 2.3 that the existence of a NTA $A_{1}^{\prime} \| A_{2}^{\prime}$ without shared clocks and such that $\psi\left(\operatorname{TTS}_{Q_{1}^{\prime}}\left(A_{1}^{\prime} \| A_{2}^{\prime}\right)\right) \sim$ $\operatorname{TTS}_{Q_{1}}\left(A_{1} \| A_{2}\right)$ is not sufficient to capture the idea that $A_{2}$ does not need to read the clocks of $A_{1}$. We are now equipped to define the relations we want to impose on the separate 

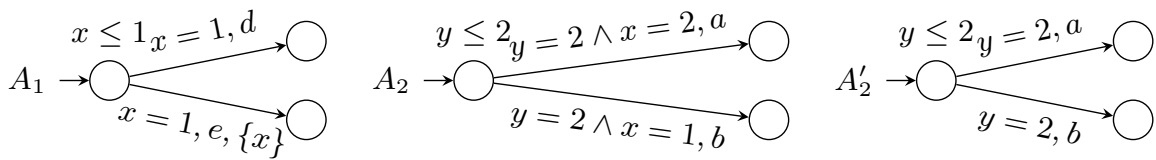

Figure 5: $A_{2}$ needs to read the clocks of $A_{1}$ and $\operatorname{TTS}_{A_{1}}\left(A_{2}\right) \sim \operatorname{TTS}_{A_{1}}\left(A_{2}^{\prime}\right)$.

components, namely $\psi\left(\operatorname{TTS}_{Q_{1}^{\prime}}\left(A_{1}^{\prime}\right)\right) \sim \operatorname{TTS}_{Q_{1}}\left(A_{1}\right)$ and $\psi\left(\operatorname{TTS}_{A_{1}^{\prime}}\left(A_{2}^{\prime}\right)\right) \sim \operatorname{TTS}_{A_{1}}\left(A_{2}\right)$. And since we have seen the importance of labeling the synchronization actions in contextual TTS by labels in $\mathbb{S} \times Q_{1}$ rather than in $\mathbb{S}$, the correspondence between the synchronization labels of $A_{1}^{\prime} \| A_{2}^{\prime}$ with those of $A_{1} \| A_{2}$ is now done by a mapping $\psi: \mathbb{S}^{\prime} \times Q_{1}^{\prime} \rightarrow \mathbb{S} \times Q_{1}$.

This settles the problem of the example of Fig. 3 where $\operatorname{TTS}_{A_{1}}\left(A_{2}^{\prime}\right) \nsim \operatorname{TTS}_{A_{1}}\left(A_{2}\right)$ (here $\left.A_{1}^{\prime}=A_{1}\right)$, but as shown in Fig. 5, a problem remains. In this example, we can see that $A_{2}$ needs to read clock $x$ of $A_{1}$ to know whether it has to perform $a$ or $b$ at time 2 , and yet $\operatorname{TTS}_{A_{1}}\left(A_{2}\right) \sim \operatorname{TTS}_{A_{1}}\left(A_{2}^{\prime}\right)$ (here also $\left.A_{1}^{\prime}=A_{1}\right)$. The intuition to understand this is that the contextual TTS merge too many states for the two systems to remain differentiable. However we remark that here, the first condition that we have required in Section 2, namely the global bisimulation between $\psi\left(\operatorname{TTS}\left(A_{1}^{\prime} \| A_{2}^{\prime}\right)\right)$ and $\operatorname{TTS}\left(A_{1} \| A_{2}\right)$, does not hold.

3.2.1. Formalization. Now we show that the conjunction of global and local bisimulations actually gives the good definition.

Definition 3.8 (Need for shared clocks). Given $A_{1} \| A_{2}$ such that $A_{1}$ does not read the clocks of $A_{2}, A_{2}$ does not need to read the clocks of $A_{1}$ iff there exists an NTA $A_{1}^{\prime} \| A_{2}^{\prime}$ without shared clocks (but with clock copies during synchronizations), using the same sets of local actions and a synchronization alphabet $\mathbb{S}^{\prime}$ related to the original one by a mapping $\psi: \mathbb{S}^{\prime} \times Q_{1}^{\prime} \rightarrow \mathbb{S} \times Q_{1}$, and such that

(1) $\psi\left(\operatorname{TTS}_{Q_{1}^{\prime}}\left(A_{1}^{\prime} \| A_{2}^{\prime}\right)\right) \sim \operatorname{TTS}_{Q_{1}}\left(A_{1} \| A_{2}\right)$ and

(2) $\psi\left(\operatorname{TTS}_{Q_{1}^{\prime}}\left(A_{1}^{\prime}\right)\right) \sim \operatorname{TTS}_{Q_{1}}\left(A_{1}\right)$ and

(3) $\psi\left(\operatorname{TTS}_{A_{1}^{\prime}}\left(A_{2}^{\prime}\right)\right) \sim \operatorname{TTS}_{A_{1}}\left(A_{2}\right)$.

Notice that this does not mean that the clock constraints that read $X_{1}$ can simply be removed from $A_{2}$ (see Fig. 2).

Lemma 3.9. When there is no restriction in $\operatorname{TTS}_{A_{1}}\left(A_{2}\right)$, any $N T A A_{1}^{\prime} \| A_{2}^{\prime}$ which has no shared clocks and which satisfies items 2 and 3 of Definition 3.8, also satisfies item 1.

Proof. When noRestriction $A_{1}\left(A_{2}\right)$ holds, then by Lemma 3.5, $\operatorname{TTS}_{Q_{1}}\left(A_{1}\right) \otimes \operatorname{TTS}_{A_{1}}\left(A_{2}\right) \approx$ $\operatorname{TTS}_{Q_{1}}\left(A_{1} \| A_{2}\right)$. So for any NTA $A_{1}^{\prime} \| A_{2}^{\prime}$ satisfying items 2 and 3 of Definition 3.8, we have $\psi\left(\operatorname{TTS}_{Q_{1}^{\prime}}\left(A_{1}^{\prime}\right)\right) \otimes \psi\left(\operatorname{TTS}_{A_{1}^{\prime}}\left(A_{2}^{\prime}\right)\right) \sim \operatorname{TTS}_{Q_{1}}\left(A_{1} \| A_{2}\right)$. It remains to show that $\psi\left(\operatorname{TTS}_{Q_{1}^{\prime}}\left(A_{1}^{\prime} \| A_{2}^{\prime}\right)\right) \approx \psi\left(\operatorname{TTS}_{Q_{1}^{\prime}}\left(A_{1}^{\prime}\right)\right) \otimes \psi\left(\operatorname{TTS}_{A_{1}^{\prime}}\left(A_{2}^{\prime}\right)\right)$. Remark that applying $\psi$ to the labels before doing the product allows more synchronizations than applying $\psi$ on the TTS of the system since $\psi$ may merge different labels. We show that, in our case, the two resulting TTS are bisimilar anyway.

For this, let $\mathcal{R}_{1}$ be a bisimulation relation between $\psi\left(\operatorname{TTS}_{Q_{1}^{\prime}}\left(A_{1}^{\prime}\right)\right)$ and $\operatorname{TTS}_{Q_{1}}\left(A_{1}\right)$, and $\mathcal{R}_{2}$ be a bisimulation relation between $\psi\left(\operatorname{TTS}_{A_{1}^{\prime}}\left(A_{2}^{\prime}\right)\right)$ and $\operatorname{TTS}_{A_{1}}\left(A_{2}\right)$. We will build inductively a bisimulation $\mathcal{R}$ between $\psi\left(\operatorname{TTS}_{Q_{1}^{\prime}}\left(A_{1}^{\prime} \| A_{2}^{\prime}\right)\right)$ and $\psi\left(\operatorname{TTS}_{Q_{1}^{\prime}}\left(A_{1}^{\prime}\right)\right) \otimes \psi\left(\operatorname{TTS}_{A_{1}^{\prime}}\left(A_{2}^{\prime}\right)\right)$ such that for any $\left(q_{1}, q_{2}\right)$ and $\left(r_{1}, r_{2}\right)$ such that $\left(q_{1}, q_{2}\right) \mathcal{R}\left(r_{1}, r_{2}\right)$, there exists a state $s_{1}$ of 
$\operatorname{TTS}_{Q_{1}}\left(A_{1}\right)$ and a state $s_{2}$ of $\operatorname{TTS}_{A_{1}}\left(A_{2}\right)$ such that $q_{1} \mathcal{R}_{1} s_{1}$ and $r_{1} \mathcal{R}_{1} s_{1}$ and $q_{2} \mathcal{R}_{2} s_{2}$ and $r_{2} \mathcal{R}_{2} s_{2}$. The inductive definition of $\mathcal{R}$ is as follows. The initial states (which are the same in both sides) are in relation; $\mathcal{R}$ is preserved by delays; $\mathcal{R}$ is preserved by playing local actions. The key is the treatment of synchronizations: when $\left(q_{1}, q_{2}\right) \mathcal{R}\left(r_{1}, r_{2}\right)$ and $q_{1} \stackrel{a_{1}}{\longrightarrow} q_{1}^{\prime}$ in $\operatorname{TTS}_{Q_{1}}\left(A_{1}\right)$ and $q_{2} \stackrel{a_{2}}{\longrightarrow} q_{2}^{\prime}$ in $\operatorname{TTS}_{A_{1}}\left(A_{2}\right)$ with $\psi\left(a_{1}\right)=\psi\left(a_{2}\right)=a$, then the existence of the $s_{1}$ and $s_{2}$ mentioned earlier ensures that there exists a state $\left(r_{1}^{\prime}, r_{2}^{\prime}\right)$ in $\psi\left(\operatorname{TTS}_{Q_{1}^{\prime}}\left(A_{1}^{\prime} \| A_{2}^{\prime}\right)\right)$ such that $\left(r_{1}, r_{2}\right) \stackrel{a}{\rightarrow}\left(r_{1}^{\prime}, r_{2}^{\prime}\right)$, and we set $\left(q_{1}^{\prime}, q_{2}^{\prime}\right) \mathcal{R}\left(r_{1}^{\prime}, r_{2}^{\prime}\right)$ for any such $\left(r_{1}^{\prime}, r_{2}^{\prime}\right)$.

3.2.2. A Criterion to Decide the Need for Shared Clocks. We are now ready to give a criterion to decide whether shared clocks are necessary.

Theorem 3.10. When there is no restriction in $\mathrm{TTS}_{A_{1}}\left(A_{2}\right)$ holds, $A_{2}$ does not need to read the clocks of $A_{1}$. When $A_{2}$ is deterministic, this condition becomes necessary.

Proof of Theorem 3.10, necessary condition when $A_{2}$ is deterministic. Like in the proof of Lemma 3.9, we show that for any NTA $A_{1}^{\prime} \| A_{2}^{\prime}$ satisfying items 2 and 3 of Definition 3.8, $\psi\left(\operatorname{TTS}_{Q_{1}^{\prime}}\left(A_{1}^{\prime} \| A_{2}^{\prime}\right)\right) \sim \operatorname{TTS}_{Q_{1}}\left(A_{1}\right) \otimes \operatorname{TTS}_{A_{1}}\left(A_{2}\right)$. But, by Lemma 3.5, when $A_{2}$ is deterministic and $\operatorname{TTS}_{A_{1}}\left(A_{2}\right)$ has restrictions, $\operatorname{TTS}_{Q_{1}}\left(A_{1}\right) \otimes \operatorname{TTS}_{A_{1}}\left(A_{2}\right)$ is not timed bisimilar to $\operatorname{TTS}_{Q_{1}}\left(A_{1} \| A_{2}\right)$ (not even weakly timed bisimilar since there are no $\varepsilon$-transitions). Hence any NTA $A_{1}^{\prime} \| A_{2}^{\prime}$ satisfying items 2 and 3 of Definition 3.8, does not satisfy item 1.

We remark from the proof that when there is a restriction in $\operatorname{TTS}_{A_{1}}\left(A_{2}\right)$, even infinite $A_{1}^{\prime}$ and $A_{2}^{\prime}$ would not help. Next section will be devoted to the constructive proof of the direct part of this theorem.

The counterexample in Fig. 4 also works here to argue that the conditions of Lemma 3.9 and Theorem 3.10 are not necessary when $A_{2}$ is not deterministic. Indeed $A_{2}^{\prime}$ with only one unguarded edge labeled by $a$ and $A_{1}^{\prime}=A_{1}$ satisfy the three items of Definition 3.8 but there is a restriction in $\operatorname{TTS}_{A_{1}}\left(A_{2}\right)$.

\section{Constructing a Network of Timed Automata without Shared Clocks}

This section is dedicated to proving Theorem 3.10 by constructing suitable $A_{1}^{\prime}$ and $A_{2}^{\prime}$. For simplicity, we assume that in $A_{2}$, the guards on the synchronizations do not read $X_{1}$. Otherwise, the constraints that read $X_{1}$ could be moved into the corresponding edges in $A_{1}$, with the intuition that, for a synchronization, each automaton can check the constraints about its own clocks.

4.1. Construction. First, our $A_{1}^{\prime}$ is obtained from $A_{1}$ by replacing all the labels $a \in \mathbb{S}$ on the synchronization edges of $A_{1}$ by $\left(a, \ell_{1}\right) \in \mathbb{S} \times L_{1}$, where $\ell_{1}$ is the output location of the edge. Therefore the synchronization alphabet between $A_{1}^{\prime}$ and $A_{2}^{\prime}$ will be $\mathbb{S}^{\prime}=\mathbb{S} \times L_{1}$, which allows $A_{1}^{\prime}$ to transmit its location after each synchronization.

Then, the idea is to build $A_{2}^{\prime}$ as a product $A_{1,2} \otimes A_{2, \bmod }$ ( $\otimes$ denotes the product of TA as it is usually defined [AD94]), where $A_{2, \bmod }$ plays the role of $A_{2}$ and $A_{1,2}$ acts as a local copy of $A_{1}^{\prime}$, from which $A_{2, \bmod }$ reads clocks instead of reading those of $A_{1}^{\prime}$. For this, as long as the automata do not synchronize, $A_{1,2}$ will evolve, simulating a run of $A_{1}^{\prime}$ that is compatible with what $A_{2}^{\prime}$ knows about $A_{1}^{\prime}$. And, as soon as $A_{1}^{\prime}$ synchronizes with $A_{2}^{\prime}$, 
$A_{2}^{\prime}$ updates $A_{1,2}$ to the actual state of $A_{1}^{\prime}$. If the clocks of $A_{1,2}$ always give the same truth value to the guards and invariants of $A_{2, \bmod }$ than the actual value of the clocks of $A_{1}^{\prime}$, then our construction behaves like $A_{1} \| A_{2}$. To check that this is the case, we equip $A_{2}^{\prime}$ with an error location, $\odot$, and edges that lead to it if there is a contradiction between the values of the clocks of $A_{1}^{\prime}$ and the values of the clocks of $A_{1,2}$. The guards of these edges are the only cases where $A_{2}^{\prime}$ reads clocks of $A_{1}^{\prime}$. Therefore, if $\odot$ is not reachable, they can be removed so that $A_{2}^{\prime}$ does not read the clocks of $A_{1}^{\prime}$. More precisely, a contradiction happens when $A_{2, \text { mod }}$ is in a given location and the guard of an outgoing edge is true according to $A_{1,2}$ and false according to $A_{1}^{\prime}$, or vice versa, or when the invariant of the current location is false according to $A_{1}^{\prime}$ (whereas it is true according to $A_{1,2}$, since $A_{2, \text { mod }}$ reads the clocks of $\left.A_{1,2}\right)$.

Namely, $\mathcal{S}_{\text {mod }}=A_{1}^{\prime} \|\left(A_{1,2} \otimes A_{2, \text { mod }}\right)$ where $A_{1,2}$ and $A_{2, \text { mod }}$ are defined as follows. $A_{1,2}=\left(L_{1}, \ell_{1}^{0}, X_{1}^{\prime}, \mathbb{S}^{\prime} \cup\{\varepsilon\}, E_{1}^{\prime}, \operatorname{Inv} v_{1}^{\prime}\right)$, where

- each clock $x^{\prime} \in X_{1}^{\prime}$ is associated with a clock $c\left(x^{\prime}\right)=x \in X_{1}$ (c is a bijection from $X_{1}^{\prime}$ to $X_{1}$ ). For any clock constraint $\gamma, \gamma^{\prime}$ denotes the clock constraint where any clock $x$ of $X_{1}$ is substituted by $x^{\prime}$ of $X_{1}^{\prime}$.

- $\forall \ell \in L_{1}, \operatorname{Inv}_{1}^{\prime}(\ell)=\operatorname{Inv}_{1}(\ell)^{\prime}$

- $E_{1}^{\prime}=\left\{\ell_{1} \stackrel{g^{\prime}, \varepsilon, r^{\prime}}{\longrightarrow} \ell_{2} \mid \exists a \in \Sigma_{1} \backslash \Sigma_{2}^{g}: \ell_{1} \stackrel{g, a, c\left(r^{\prime}\right)}{\longrightarrow} \ell_{2} \in E_{1}\right\}$

(simulate local actions of $A_{1}$ )

$\cup \quad\left\{\ell \stackrel{t t,\left(a, \ell_{2}\right), c}{\longrightarrow} \ell_{2} \mid \ell \in L_{1} \wedge a \in \mathbb{S} \wedge \exists \ell_{1} \stackrel{g, a, r}{\longrightarrow} \ell_{2} \in E_{1}\right\}$

(update the state of $A_{1,2}$ at each synchronization with $A_{1}$ )

where $c$ denotes the assignment of any clock $x^{\prime} \in X_{1}^{\prime}$ with the value of its associated clock $c\left(x^{\prime}\right)=x \in X_{1}$ (written $x^{\prime}:=x$ in Fig. 6).

$A_{2, \text { mod }}=\left(L_{2} \cup\{\odot\}, \ell_{2}^{0}, X_{2} \cup X_{1}^{\prime} \cup X_{1},\left(\Sigma_{2} \backslash \Sigma_{1}\right) \cup \mathbb{S}^{\prime}, E_{2}^{\prime}, I n v_{2}^{\prime}\right)$, where

- $\forall \ell \in L_{2}, \operatorname{Inv}_{2}^{\prime}(\ell)=\operatorname{Inv}_{2}(\ell)^{\prime}$ and $\operatorname{Inv}_{2}^{\prime}(\odot)=\boldsymbol{t}$,

- $E_{2}^{\prime}=\left\{\ell_{1} \stackrel{g^{\prime}, a, r}{\longrightarrow} \ell_{2} \mid \ell_{1} \stackrel{g, a, r}{\longrightarrow} \ell_{2} \in E_{2} \wedge a \notin \mathbb{S}\right\}$

$\cup \quad\left\{\ell_{1} \stackrel{g,(a, \ell), r}{\longrightarrow} \ell_{2} \mid \ell_{1} \stackrel{g, a, r}{\longrightarrow} \ell_{2} \in E_{2} \wedge a \in \mathbb{S} \wedge \ell \in L_{1}\right\}$

$\cup \quad\left\{\ell \stackrel{\neg I n v_{2}(\ell), \varepsilon, \emptyset}{\longrightarrow} \odot \mid \ell \in L_{2}\right\}$

$\cup \quad\left\{\ell \stackrel{g^{\prime} \wedge \neg g, \varepsilon, \emptyset}{\longrightarrow} \odot \mid \ell \stackrel{g, a, r}{\longrightarrow} \ell^{\prime} \in E_{2} \wedge a \notin \mathbb{S}\right\}$

$\cup \quad\left\{\ell \stackrel{\neg g^{\prime} \wedge g, \varepsilon, \emptyset}{\longrightarrow} \odot \mid \ell \stackrel{g, a, r}{\longrightarrow} \ell^{\prime} \in E_{2} \wedge a \notin \mathbb{S}\right\}$.

For the example of Fig. 2, $A_{1,2}$ and $A_{2, \text { mod }}$ are pictured in Fig. 6 .

We now prove the correspondence between a state of $\mathcal{S}_{\text {mod }}$ and two states of $\operatorname{TTS}\left(A_{1} \|\right.$ $\left.A_{2}\right)$ that are merged into the same state of $\operatorname{TTS}_{A_{1}}\left(A_{2}\right)$. This is stated in the following proposition. A state of $\mathcal{S}_{\text {mod }}$ is denoted as $\left(s_{1}, s_{1,2}, s_{2}\right)=\left(\left(\ell_{1}, v_{\mid X_{1}}\right),\left(\ell_{1,2}, v_{\mid X_{1}^{\prime}}\right),\left(\ell_{2}, v_{\mid X_{2} \backslash X_{1}}\right)\right)$. For a given state of $A_{1,2}, s_{1,2}=\left(\ell_{1,2}, v_{\mid X_{1}^{\prime}}\right)$, we denote by $s_{1,2}^{\prime}$ the state $\left(\ell_{1,2}, v^{\prime}\right)$, where $v^{\prime}: X_{1} \rightarrow \mathbb{R}_{\geq 0}$ is defined as: for any $x \in X_{1}, v^{\prime}(x)=v\left(x^{\prime}\right)$ (i.e. $s_{1,2}^{\prime}$ is a state of $A_{1}$ ). Reciprocally, for a given state of $A_{1}, s_{1,2}^{\prime}=\left(\ell_{1,2}, v^{\prime}\right), s_{1,2}$ denotes the state $\left(\ell_{1,2}, v\right)$, where $v: X_{1}^{\prime} \rightarrow \mathbb{R}_{\geq 0}$ is defined as: for any $x^{\prime} \in X_{1}^{\prime}, v\left(x^{\prime}\right)=v^{\prime}(x)$.

Proposition 4.1. Let $\left(s_{1}, s_{1,2}, s_{2}\right)$ be a state of $\mathcal{S}_{\text {mod }}$. If along one path that leads to $\left(s_{1}, s_{1,2}, s_{2}\right)$ no edge leading to $\odot$ is enabled, then there exists $S_{1}$ such that $\left(S_{1}, s_{2}\right)$ is a reachable state of $\operatorname{TTS}_{A_{1}}\left(A_{2}\right)$ and $s_{1}$ and $s_{1,2}^{\prime}$ are both in $S_{1}$.

Conversely, let $\left(S_{1}, s_{2}\right)$ be a reachable state of $\operatorname{TTS}_{A_{1}}\left(A_{2}\right)$, and $s_{1}$ and $s_{1,2}^{\prime}$ be some states in $S_{1}$. Then $\left(s_{1}, s_{1,2}, s_{2}\right)$ is a state of $\mathcal{S}_{\text {mod }}$. 

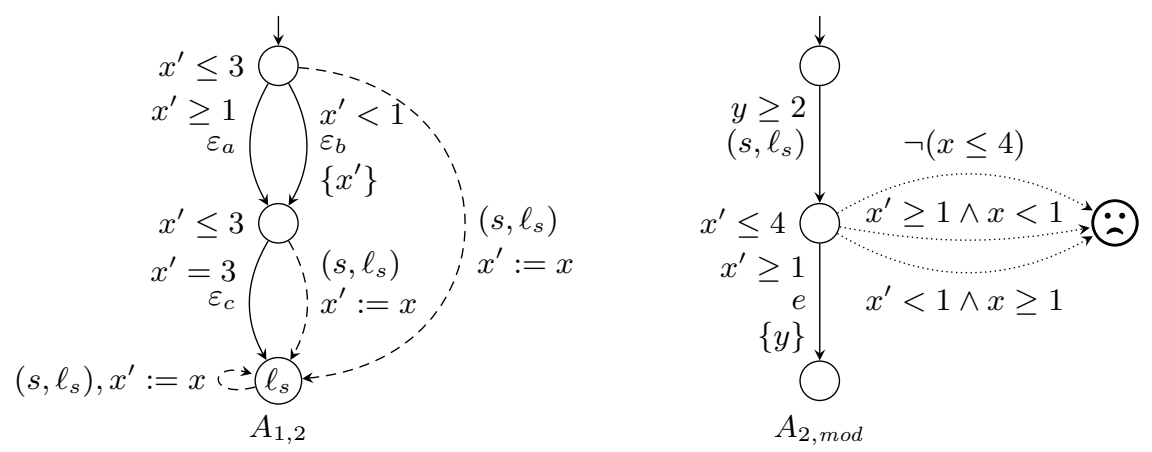

Figure 6: $A_{1,2}$ and $A_{2, \bmod }$ for the example of Fig. 2. We represent by dotted arcs the edges leading to the error state, and by dashed arcs those used during synchronizations to reset $A_{1,2}$ to the actual state of $A_{1}$.

Proof. Let $\left(s_{1}, s_{1,2}, s_{2}\right)$ be a reachable state of $\mathcal{S}_{\text {mod }}$, such that there is a path $\rho$ from the initial state $\left(s_{1}^{0}, s_{1,2}^{0}, s_{2}^{0}\right)$ to $\left(s_{1}, s_{1,2}, s_{2}\right)$ that does not enable any edges leading to $(*)$ (except maybe from $\left.\left(s_{1}, s_{1,2}, s_{2}\right)\right)$. We give a recursive proof. First, for the initial state $\left(s_{1}^{0}, s_{1,2}^{0}, s_{2}^{0}\right)$ of $\mathcal{S}_{\text {mod }}, s_{1}^{0}$ and $s_{1,2}^{0 \prime}$ are both in $S_{1}^{0}$ such that $\left(S_{1}^{0}, s_{2}^{0}\right)$ is the initial state of $\operatorname{TTS}_{A_{1}}\left(A_{2}\right)$. Now, assume this is true for some $\left(p_{1}, p_{1,2}, p_{2}\right)$ visited along $\rho$. That is, there exists $P_{1}$ such that $\left(P_{1}, p_{2}\right)$ is reachable and $p_{1}, p_{1,2}^{\prime} \in P_{1}$. Then, the next state $s^{\prime}$ visited along $\rho$ is reached after one of the following steps:

- local action in $A_{1}^{\prime}: s^{\prime}=\left(q_{1}, p_{1,2}, p_{2}\right)$ such that $q_{1} \in \mathrm{UR}\left(p_{1}\right) \subseteq P_{1}$,

- local action in $A_{1,2}: s^{\prime}=\left(p_{1}, q_{1,2}, p_{2}\right)$ such that $q_{1,2}^{\prime} \in \mathrm{UR}\left(p_{1,2}^{\prime}\right) \subseteq P_{1}$,

- local action in $A_{2}: s^{\prime}=\left(p_{1}, p_{1,2}, q_{2}\right)$ such that there exists $S_{1}^{\prime}$ such that $\left(S_{1}^{\prime}, q_{2}\right)$ is reachable from $\left(P_{1}, q_{2}\right)$ by the same action, and, since no edge leading to $\odot$ is enabled, both $\left(p_{1}, p_{2}\right)$ and $\left(p_{1,2}^{\prime}, p_{2}\right)$ enable this step in $\operatorname{TTS}\left(A_{1} \| A_{2}\right)$. Therefore, $p_{1}, p_{1,2}^{\prime} \in S_{1}^{\prime}$.

- synchronization: $s^{\prime}=\left(q_{1}, q_{1,2}, q_{2}\right)$ such that there exists $S_{1}^{\prime}=\operatorname{UR}\left(q_{1}\right)$ such that $\left(S_{1}^{\prime}, q_{2}\right)$ is reachable from $\left(P_{1}, q_{2}\right)$ by the same action, and $q_{1}=q_{1,2}^{\prime} \in S_{1}^{\prime}$.

By recursion, $\left(s_{1}, s_{1,2}, s_{2}\right)$ also satisfies the property, that is, there exists $S_{1}$ such that $\left(S_{1}, s_{2}\right)$ is reachable and $s_{1}, s_{1,2}^{\prime} \in S_{1}$.

Conversely, let denote by $P\left(S_{1}, s_{2}\right)$ the fact that for any reachable state $\left(S_{1}, s_{2}\right)$ of $\operatorname{TTS}_{A_{1}}\left(A_{2}\right)$, for any states $s_{1}, s_{1,2}^{\prime} \in S_{1},\left(s_{1}, s_{1,2}, s_{2}\right)$ is a reachable state of $\mathcal{S}_{\text {mod }}$. First, for any $s_{1}, s_{1,2}^{\prime} \in S_{1}^{0}=\mathrm{UR}\left(s_{1}^{0}\right),\left(s_{1}, s_{1,2}, s_{2}^{0}\right)$ is a reachable state, because by construction, $A_{1,2}$ can only mimic (as long as there is no synchronization) one possible behavior of $A_{1}$ to reach $s_{1,2}$ from $s_{1}^{0}$, therefore $P\left(S_{1}^{0}, s_{2}^{0}\right)$ holds. Assume that for some reachable state $\left(S_{1}, s_{2}\right)$ $P\left(S_{1}, s_{2}\right)$ holds. Then any state reachable in one step from $\left(S_{1}, s_{2}\right)$ is reached by one of the following steps.

- If for some $a \in \Sigma_{2} \backslash \mathbb{S},\left(S_{1}, s_{2}\right) \stackrel{a}{\rightarrow}\left(S_{1}^{\prime}, s_{2}^{\prime}\right)$, then for any $s_{1}, s_{1,2}^{\prime} \in S_{1}^{\prime} \subseteq S_{1},\left(s_{1}, s_{1,2}^{\prime}, s_{2}\right) \stackrel{a}{\rightarrow}$ $\left(s_{1}, s_{1,2}^{\prime}, s_{2}^{\prime}\right)$, i.e. $P\left(S_{1}^{\prime}, s_{2}^{\prime}\right)$ holds.

- If for some $\left(a, s_{1}^{\prime}\right) \in \mathbb{S} \times Q_{1},\left(S_{1}, s_{2}\right) \stackrel{a, s_{1}^{\prime}}{\longrightarrow}\left(S_{1}^{\prime}, s_{2}^{\prime}\right)$, then $S_{1}^{\prime}=\mathrm{UR}\left(s_{1}^{\prime}\right)$, and for any $s_{1}, s_{1,2}^{\prime} \in S_{1}^{\prime},\left(s_{1}, s_{1,2}, s_{2}^{\prime}\right)$ can be reached from some $\left(p_{1}, p_{1,2}, s_{2}\right)$ such that $p_{1}, p_{1,2}^{\prime} \in S_{1}$. Indeed, in $\mathcal{S}_{\text {mod }}$, synchronization $\left(\left(a, \ell_{1}^{\prime}\right), s_{1}^{\prime}\right)$ resets $A_{1,2}$ in the same state as $A_{1}$ and then 
$A_{1}$ performs some local actions while $A_{1,2}$ also performs some local actions mimicking one possible behavior of $A_{1}$ (that is why $s_{1,2}^{\prime} \in S_{1}^{\prime}$ ). Hence $P\left(S_{1}^{\prime}, s_{2}^{\prime}\right)$ holds.

- If for some $d \in \mathbb{R}_{\geq 0},\left(S_{1}, s_{2}\right) \stackrel{d}{\rightarrow}\left(S_{1}^{\prime}, s_{2}^{\prime}\right)$, then we use the same reasoning as for a synchronization. Since $A_{1,2}$ is built so that it mimics any possible behavior of $A_{1}$ between synchronizations, any state $s_{1,2}^{\prime} \in S_{1}^{\prime}$ reachable by $A_{1}$ during this delay corresponds to a state $s_{1,2}$ reachable by $A_{1,2}$. Hence $P\left(S_{1}^{\prime}, s_{2}^{\prime}\right)$ also holds.

By recursion, $P\left(S_{1}, s_{2}\right)$ holds for any reachable state $\left(S_{1}, s_{2}\right)$.

Lastly, the following lemma will be used to prove the direct part of Theorem 3.10 .

Lemma 4.2. $:$ is reachable in $\mathcal{S}_{\text {mod }}$ iff there is a restriction in $\operatorname{TTS}_{A_{1}}\left(A_{2}\right)$.

Proof. Assume $:$ is not reachable in $\mathcal{S}_{\text {mod }}$. From Proposition 4.1, we know that for any state $\left(S_{1}, s_{2}\right)$ of $\operatorname{TTS}_{A_{1}}\left(A_{2}\right)$, for any $s_{1}, s_{1,2}^{\prime}$ in $S_{1}$, there is a corresponding state $s=$ $\left(\left(\ell_{1}, v_{\mid X_{1}}\right),\left(\ell_{1,2}, v_{\mid X_{1}^{\prime}}\right),\left(\ell_{2}, v_{\mid X_{2} \backslash X_{1}}\right)\right)=\left(s_{1}, s_{1,2}, s_{2}\right)$ of $\mathcal{S}_{\text {mod }}$. Moreover, for any such $s$, if there is an outgoing edge towards $:$ from $\ell_{2}$, then this edge is never enabled. That is, for any time constraint $\gamma$ read in $\ell_{2}$ in the original system $\mathcal{S}$ (invariant of $\ell_{2}$ or guard of an outgoing edge with a local action), $v_{\mid X_{2} \cup X_{1}} \models \gamma \Longleftrightarrow v_{\mid\left(X_{2} \backslash X_{1}\right) \cup X_{1}^{\prime}} \models \gamma^{\prime}$. Hence for any enabled step from $\left(S_{1}, s_{2}\right), s_{1}$ and $s_{1,2}^{\prime}$ are in the same restriction. Therefore, noRestriction $_{A_{1}}\left(A_{2}\right)$.

Assume $(-)$ is reachable in $\mathcal{S}_{\text {mod }}$. From Proposition 4.1, we know that for any state $s=$ $\left(\left(\ell_{1}, v_{\mid X_{1}}\right),\left(\ell_{1,2}, v_{\mid X_{1}^{\prime}}\right),\left(\ell_{2}, v_{\mid X_{2} \backslash X_{1}}\right)\right)=\left(s_{1}, s_{1,2}, s_{2}\right)$ of $\mathcal{S}_{\text {mod }}$, reached after a path that does not enable edges leading to $\odot$ (except maybe from this last state), there is a corresponding state $\left(S_{1}, s_{2}\right)$ of $\operatorname{TTS}_{A_{1}}\left(A_{2}\right)$ such that $s_{1}$ and $s_{1,2}^{\prime}$ are both in $S_{1}$. If $\odot$ can be reached, then consider a path that reaches $\odot$ and such that no edge leading to $\odot$ was enabled before along the path. The last state $s$ of $\mathcal{S}_{\text {mod }}$ visited before $\odot$ is such that for some time constraint $\gamma$ evaluated at $s$ from $\ell_{2}, v_{\mid X_{2} \cup X_{1}} \models \gamma$ and $v_{\mid\left(X_{2} \backslash X_{1}\right) \cup X_{1}^{\prime}} \not \models \gamma^{\prime}$ (or conversely). Therefore, a local action or local delay is possible from $\left(s_{1}, s_{2}\right)$ and not from $\left(s_{1,2}^{\prime}, s_{2}\right)$. Hence $\left(S_{1}, s_{2}\right)$ is a state with a restriction.

We now give a first simple case for which Theorem 3.10 can be proved easily. We say that $A_{1}$ has no urgent synchronization if for any location, when the invariant reaches its limit, a local action is enabled. Under this assumption, we can show that $A_{2}^{\prime}=A_{1,2} \otimes A_{2, \text { mod }}^{\prime}$, where $A_{2, \text { mod }}^{\prime}$ is $A_{2, \text { mod }}$ without location $:$ (that is never reached according to Lemma 4.2) and its ingoing edges, is suitable. Indeed, we can show that $A_{2}^{\prime}$ does not read $X_{1}$ and is such that $\psi\left(\operatorname{TTS}_{A_{1}^{\prime}}\left(A_{2}^{\prime}\right)\right) \sim \operatorname{TTS}_{A_{1}}\left(A_{2}\right)$, where for any $\left(\left(a, \ell_{1}\right), s_{1}\right) \in \mathbb{S}^{\prime} \times Q_{1}^{\prime}, \psi\left(\left(\left(a, \ell_{1}\right), s_{1}\right)\right)=\left(a, s_{1}\right)$. Obviously, item 2 of Definition 3.8 holds, and Lemma 3.9 says that item 1 also holds.

When $A_{1}$ has urgent synchronizations, this construction allows one to check the absence of restriction in $\operatorname{TTS}_{A_{1}}\left(A_{2}\right)$, but it does not give directly a suitable $A_{2}^{\prime}$. We define the construction of $A_{2}^{\prime}$ for the general case in Subsection 4.3 .

Proof of Theorem 3.10, direct part, when no urgent synchronization in $A_{1}$. Assume noRestriction $A_{1}\left(A_{2}\right)$. We consider $A_{2}^{\prime}=A_{1,2} \otimes A_{2, \bmod }^{\prime}$ where $A_{2, \bmod }^{\prime}$ is $A_{2, \bmod }$ without $(:)$ (that is never reached according to Lemma 4.2) and its ingoing edges. Therefore, $A_{2, \text { mod }}^{\prime}$ does not read $X_{1}$ and neither does $A_{2}^{\prime}=A_{1,2} \otimes A_{2, \text { mod }}^{\prime}$. Below we show that $A_{2}^{\prime}$ is a suitable candidate because $\psi\left(\operatorname{TTS}_{A_{1}^{\prime}}\left(A_{2}^{\prime}\right)\right) \sim \operatorname{TTS}_{A_{1}}\left(A_{2}\right)\left(\psi\left(\operatorname{TTS}_{Q_{1}^{\prime}}\left(A_{1}^{\prime}\right)\right) \sim \operatorname{TTS}_{Q_{1}}\left(A_{1}\right)\right.$ obviously holds). 
Let $\mathcal{R}$ be the relation such that for any reachable state $\left(S_{1}, s_{2}\right)$ of $\operatorname{TTS}_{A_{1}}\left(A_{2}\right)$, and any reachable state $\left(S_{1}^{\prime}, s_{2}^{\prime}\right)$ of $\psi\left(\operatorname{TTS}_{A_{1}^{\prime}}\left(A_{2}^{\prime}\right)\right)$,

$$
\left(S_{1}, s_{2}\right) \mathcal{R}\left(S_{1}^{\prime}, s_{2}^{\prime}\right) \stackrel{\text { def }}{\Longleftrightarrow}\left\{\begin{array}{l}
s_{2}=\left(\ell_{2}, v_{2}\right) \text { and } s_{2}^{\prime}=\left(\left(\ell_{1,2}, \ell_{2}\right), v_{2}^{\prime}\right) \text { s.t. } \\
\forall x \in X_{2} \backslash X_{1}, v_{2}(x)=v_{2}^{\prime}(x) \\
S_{1}=S_{1}^{\prime}
\end{array}\right.
$$

i.e. $A_{2}$ and $A_{2, \text { mod }}^{\prime}$ are both in $\ell_{2}$ and their local clocks have the same value, and $A_{1}$ and $A_{1}^{\prime}$ are in indistinguishable states (states merged in a same contextual state $S_{1}$ ). Obviously, the initial states, $\left(S_{1}^{0}, s_{2}^{0}\right)$ and $\left(S_{1}^{0}, s_{2}^{0 \prime}\right)$, are $\mathcal{R}$-related. Since there is no marked state in $\operatorname{TTS}_{A_{1}}\left(A_{2}\right)$ (resp. in $\operatorname{TTS}_{A_{1}^{\prime}}\left(A_{2}^{\prime}\right)$ ), for any state $s=\left(S_{1}, s_{2}\right)$ (resp. $s^{\prime}=\left(S_{1}^{\prime}, s_{2}^{\prime}\right)$ ) of this TTS, all time constraints read by automaton 2 in $\ell_{2}$ (invariant of $\ell_{2}$ and guards of the outgoing edges) have the same truth value for all the states $\left(s_{1}, s_{2}\right)$ such that $s_{1} \in S_{1}$ (resp. $s_{1} \in S_{1}^{\prime}$ ). In the sequel, we say that valuation $V$ of $s$ (resp. $V^{\prime}$ of $s^{\prime}$ ) satisfies constraint $g$, when the valuations of all states $\left(s_{1}, s_{2}\right)$ in $s$ (resp. in $s^{\prime}$ ) satisfy $g$. Assume now that for some reachable states $\left(S_{1}, s_{2}\right)$ and $\left(S_{1}^{\prime}, s_{2}^{\prime}\right),\left(S_{1}, s_{2}\right) \mathcal{R}\left(S_{1}^{\prime}, s_{2}^{\prime}\right)$.

Local Action. If $a \in \Sigma_{2} \backslash \Sigma_{1}$ is enabled from $\left(S_{1}, s_{2}\right)$, then, there is an associated edge in $A_{2}$, $\ell_{2} \stackrel{g, a, r}{\longrightarrow} p_{2}$ such that guard $g$ is satisfied by $V$. Let $g^{\prime}$ be the guard on the corresponding outgoing edge $\left(\ell_{1,2}, \ell_{2}\right) \stackrel{g^{\prime}, a, r}{\longrightarrow}\left(\ell_{1,2}, p_{2}\right)$ in $A_{2}^{\prime} . g$ uses clocks in $X_{2}$, and by construction, $g^{\prime}$ has the same form but with clocks in $\left(X_{2} \backslash X_{1}\right) \uplus X_{1}^{\prime}$. $\left(S_{1}, s_{2}\right) \mathcal{R}\left(S_{1}^{\prime}, s_{2}^{\prime}\right)$ says that $v_{2}$ and $v_{2}^{\prime}$ coincide on $X_{2} \backslash X_{1}$, and since () is never reached in $\mathcal{S}_{m o d}, V$ satisfies the constraints of $g$ on $X_{1}$ iff $V^{\prime}$ satisfies the constraints of $g^{\prime}$ on $X_{1}^{\prime}$. That is, $V \models g \Longleftrightarrow V^{\prime} \models g^{\prime}$. Therefore $A_{2}^{\prime}$ can also perform $a$ from $\left(S_{1}, s_{2}^{\prime}\right)$ and the states reached in both systems are $\mathcal{R}$-related: $\left(S_{1}, q_{2}\right) \mathcal{R}\left(S_{1}, q_{2}^{\prime}\right)$, because $q_{2}=\left(p_{2}, v_{2}[r]\right)$ and $q_{2}^{\prime}=\left(\left(\ell_{1,2}, p_{2}\right), v_{2}^{\prime}[r]\right)$. This also holds reciprocally.

Synchronization. Assume for some $\left(a, s_{1}^{\prime}\right) \in \mathbb{S} \times Q_{1},\left(S_{1}, s_{2}\right) \stackrel{a, s_{1}^{\prime}}{\longrightarrow}\left(S_{1}^{\prime}, q_{2}\right)$. That is, there is an edge $\ell_{2} \stackrel{g_{2}, a, r_{2}}{\longrightarrow} p_{2}$ in $A_{2}$ such that $v_{2} \models g_{2}$ and $q_{2}=\left(p_{2}, v_{2}\left[r_{2}\right]\right)$ and, for some $\left(\ell_{1}, v_{1}\right) \in S_{1}$, an edge $\ell_{1} \stackrel{g_{1}, a, r_{1}}{\longrightarrow} p_{1}$ in $A_{1}$ such that $v_{1} \models g_{1}$ and $s_{1}^{\prime}=\left(p_{1}, v_{1}\left[r_{1}\right]\right) \in S_{1}^{\prime}$. Hence, synchronization $\left(\left(a, p_{1}\right), s_{1}^{\prime}\right)$ is also enabled from state $\left(S_{1}, s_{2}^{\prime}\right)$ because $A_{2, \text { mod }}$ is in the same location as $A_{2}$, and has the same clock values over $X_{2} \backslash X_{1}$, and $A_{1}^{\prime}$ is also in some state of $S_{1}$, therefore, there is also the same state $\left(\ell_{1}, v_{1}\right) \in S_{1}$ which enables $\left(a, p_{1}\right)$. We do not consider $A_{1,2}$ because it is always ready to synchronize. Moreover, the state reached in $\psi\left(\operatorname{TTS}_{A_{1}^{\prime}}\left(A_{2}^{\prime}\right)\right)$ after this synchronization is $\left(S_{1}^{\prime}, q_{2}^{\prime}\right)$ such that $\left(S_{1}^{\prime}, q_{2}\right) \mathcal{R}\left(S_{1}^{\prime}, q_{2}^{\prime}\right)$, because $q_{2}=\left(p_{2}, v_{2}\left[r_{2}\right]\right)$ and $q_{2}^{\prime}=\left(\left(p_{1,2}, p_{2}\right),\left(v_{2}^{\prime}\left[r_{2}\right]\right)[c]\right)$ where $c$ denotes the copy of the clocks of $X_{1}$ into their associated clocks of $X_{1}^{\prime}$ and therefore $c$ modifies only clocks that we do not consider in relation $\mathcal{R}$, and $r_{2} \subseteq C_{2} \subseteq\left(X_{2} \backslash X_{1}\right)$ resets the same clocks in both systems. And reciprocally.

Local Delay. Assume for some $d \in \mathbb{R}_{\geq 0},\left(S_{1}, s_{2}\right) \stackrel{d}{\rightarrow}\left(S_{1}^{\prime}, q_{2}\right)$. Then, $V+d \models \operatorname{Inv}_{2}\left(\ell_{2}\right)$, and since $\odot$ is never reached in $\mathcal{S}_{\text {mod }}, V+d \models \operatorname{Inv}_{2}\left(\ell_{2}\right) \Longleftrightarrow V^{\prime}+d \models \operatorname{Inv}_{2}^{\prime}\left(\ell_{2}\right)$. That is, the same delay is enabled from $\left(S_{1}, s_{2}^{\prime}\right)$ while $A_{1,2}$ may perform some local steps: $\left(S_{1}, s_{2}^{\prime}\right)\left(\stackrel{g_{0}, \varepsilon, r_{0}}{\longrightarrow}\right)^{*} \stackrel{d_{0}}{\longrightarrow}$ $\left(\stackrel{g_{n}, \varepsilon, r_{n}}{\longrightarrow}\right)^{*} \ldots \stackrel{d_{n}}{\longrightarrow}\left(S_{1}^{\prime \prime}, q_{2}^{\prime}\right)$, where $\sum_{i=0}^{n} d_{i}=d, g_{i}$ is a guard over $X_{1}^{\prime}$ and $r_{i}$ is a reset included 
in $X_{1}^{\prime}$. This works because we assumed that $A_{1}$ has no urgent synchronization (and so does $\left.A_{1}^{\prime}\right)$. Therefore, $A_{1,2}$ cannot force a synchronization.

Reciprocally, if we can perform a delay $d$ from $\left(S_{1}, s_{2}^{\prime}\right)$, then $V^{\prime}+d \models \operatorname{Inv}_{2}^{\prime}\left(\ell_{2}\right) \wedge$ $\operatorname{Inv} v_{1}^{\prime}\left(\ell_{1,2}\right)$. And since $V+d \models \operatorname{Inv}_{2}\left(\ell_{2}\right) \Longleftrightarrow V^{\prime}+d \models \operatorname{Inv} v_{2}^{\prime}\left(\ell_{2}\right)$, we can perform the same delay from $\left(S_{1}, s_{2}\right)$.

Moreover, we reach equivalent states in both systems. Indeed, $A_{2}$ and $A_{2, \text { mod }}^{\prime}$ stay in the same location, the clocks in $X_{2} \backslash X_{1}$ increase their value by $d$, and the set of states of $A_{1}$ and $A_{1}^{\prime}$ becomes $S_{1}^{\prime}=S_{1}^{\prime \prime}=\left\{s_{1}^{\prime} \mid \exists s_{1} \in S_{1}, \rho \in \operatorname{Paths}\left(\Sigma_{1} \backslash \Sigma_{2}^{q}, d\right):\left(s_{1}, s_{2}\right) \stackrel{\rho}{\Rightarrow}\left(s_{1}^{\prime}, q_{2}\right)\right\}$.

Therefore, $\mathcal{R}$ is a weak timed bisimulation and $\psi\left(\operatorname{TTS}_{A_{1}^{\prime}}\left(A_{2}^{\prime}\right)\right) \sim \operatorname{TTS}_{A_{1}}\left(A_{2}\right)$. Lastly, by Lemma 3.9. $\psi\left(\operatorname{TTS}_{Q_{1}^{\prime}}\left(A_{1}^{\prime} \| A_{2}^{\prime}\right)\right) \sim \operatorname{TTS}_{Q_{1}}\left(A_{1} \| A_{2}\right)$ also, and $A_{2}$ does not need to read $X_{1}$.

In the example of Fig. 2, $(;)$ is not reachable in $\mathcal{S}_{\text {mod }}$ (see Fig. 6), therefore $A_{2}$ does not need to read $X_{1}$. For an example where $\odot$ is reachable, consider the same example

with an additional edge $\stackrel{t t, f,\{x\}}{\longrightarrow}$ from the end location of $A_{1}$ to a new location. Location (2) can now be reached in $\mathcal{S}_{\text {mod }}$, for example consider a run where $s$ is performed at time 2 leading to a state where $v(x)=2$ and $v\left(x^{\prime}\right)=2$, and then $A_{1}$ immediately performs $f$ and resets $x$, leading to a state where the valuation $v^{\prime}$ is such that $v^{\prime}(x)=0$ and $v^{\prime}\left(x^{\prime}\right)=2$, and satisfies guard $x^{\prime} \geq 1 \wedge x<1$ in $\mathcal{S}_{\text {mod }}$. Therefore, with this additional edge in $A_{1}, A_{2}$ needs to read $X_{1}$. Indeed, without this edge, $A_{2}$ knows that $A_{1}$ cannot modify $x$ after the synchronization, but with this edge, $A_{2}$ does not know whether $A_{1}$ has performed $f$ and reset $x$, while this may change the truth value of its guard $x \geq 1$.

\subsection{Complexity.}

PSPACE-hardness. The reachability problem for timed automata is known to be PSPACEcomplete AD90. We will reduce this problem to our problem of deciding whether $A_{2}$ needs to read the clocks of $A_{1}$. Consider a timed automaton $A$ over alphabet $\Sigma$, with some location $\ell$. Build the timed automaton $A_{2}$ as $A$ augmented with two new locations $\ell^{\prime}$ and $\ell^{\prime \prime}$ and two edges, $\ell \stackrel{t t, \varepsilon, \emptyset}{\longrightarrow} \ell^{\prime}$ and $\ell^{\prime} \stackrel{x=1, a, \emptyset}{\longrightarrow} \ell^{\prime \prime}$, where $x$ is a fresh clock, and $a$ is some action in $\Sigma$. Let $A_{1}$ be the one of Fig. 4 with an action $b \notin \Sigma$. Then, $\ell$ is reachable in $A$ iff $A_{2}$ needs to read $x$ which belongs to $A_{1}$. Therefore the problem of deciding whether $A_{2}$ needs to read the clocks of $A_{1}$ is also PSPACE-hard.

PSPACE-membership. Moreover, we can show that when $A_{2}$ is deterministic, our problem is in PSPACE. Indeed, by Theorem 3.10 and Lemma 4.2 , $\odot$ is not reachable iff noRestriction $_{A_{1}}\left(A_{2}\right)$ iff $A_{2}$ does not need to read the clocks of $A_{1}$. Since the size of the modified system on which we check the reachability of $\odot$ is polynomial in the size of the original system, our problem is in PSPACE. 

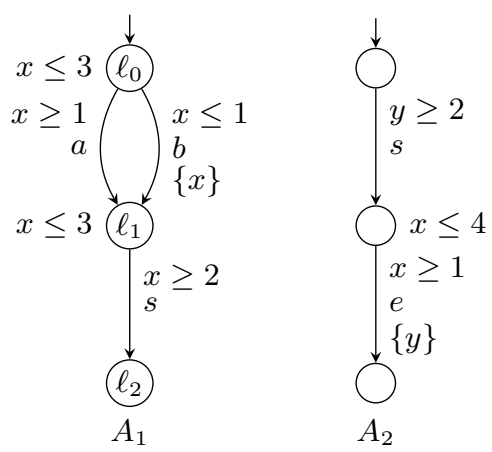

Figure 7: $A_{1}$ has an urgent synchronization.

4.3. Dealing with Urgent Synchronizations. If we use exactly the same construction as before and allow urgent synchronizations, the following problem may occur. Remind that $A_{1,2}$ simulates a possible run of $A_{1}^{\prime}$ while $A_{1}^{\prime}$ plays its actual run. There is no reason why the two runs should coincide. Thus it may happen that the run simulated by $A_{1,2}$ reaches a state where the invariant expires and only a synchronization is possible. Then $A_{2}^{\prime}$ is expecting a synchronization with $A_{1}^{\prime}$, but it is possible that the actual $A_{1}^{\prime}$ has not reached a state that enables this synchronization. Intuitively, $A_{2}^{\prime}$ should then realize that the simulated run cannot be the actual one and try another run compatible with the absence of synchronization.

In fact, between two synchronizations, $A_{1,2}$, the local copy of $A_{1}$, can be constructed to simulate only one fixed run of $A_{1}$, instead of being able to simulate all its runs. If this run is well chosen, then the situation described above never happens, and we can use a construction similar to the one above, on which we can prove that if $\odot$ is not reachable, then any run of $A_{1}$ is compatible with the fixed run of $A_{1,2}$, and $A_{2}$ can avoid reading the clocks of $A_{1}$.

Therefore, the idea of the construction is to force $A_{1,2}$ to simulate one of the runs of $A_{1}$ (from the state reached after the last synchronization) that has maximal duration before it synchronizes again with $A_{2, \text { mod }}$ (or never synchronizes again if possible). There may not be any such run if some time constraints are strict inequalities, but the idea can be adapted even to this case. This choice of a run of $A_{1}$ is as valid as the others, and it prevents the system from having to deal with the subtle situation that we described above. Below, we describe the construction of $A_{1,2}$ in two cases:

(1) After any synchronization there is a local run of maximal duration.

(2) It may happen that, after a synchronization, there is no run of maximal duration because of some strict time constraints.

Case 1: After any synchronization there is a local run of maximal duration. Consider automaton $A_{1}$ in Fig. 17. We can see that, for the urgent synchronization to happen as late as possible, $A_{1,2}$ has to fire $b$ at time 1 , so that it can then wait 3 time units before synchronizing, although it is still able to synchronize at any time (we add the same dashed edges as in Fig. 6). Fig. 8 shows a timed automaton that achieves the desired behaviour for $A_{1,2}$ using a fresh clock $z$ to force the simulation of $b$ at time 1 . 


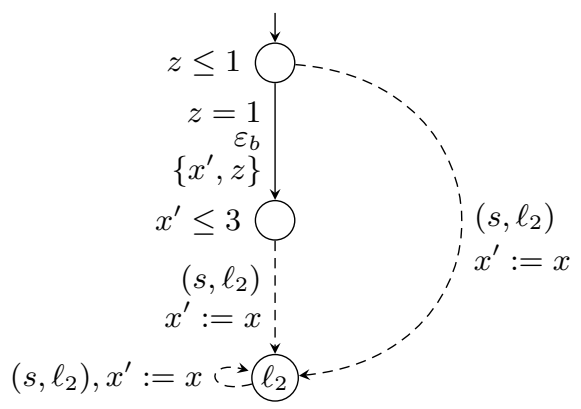

Figure 8: $A_{1,2}$ associated with $A_{1}$ of Fig. 7 .

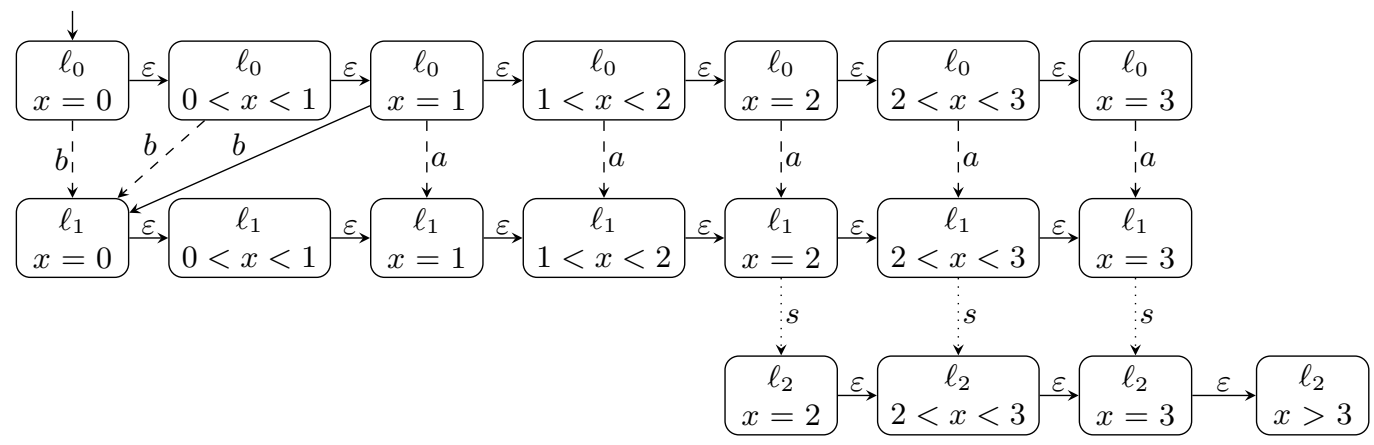

Figure 9: The region automaton of $A_{1}$ of Fig. 7, The dashed arcs indicate occurrences of internal actions of $A_{1}$ that will be removed in the construction of $A_{1,2}$ in order to force a run of maximal duration between synchronizations. The occurrences of the synchronization $s$, represented by dotted arrows, are treated separately in the construction of $A_{1,2}$.

This can be generalized for any $A_{1}$. The idea is essentially to force $A_{1,2}$ to follow the appropriate finite or ultimately periodic path in the region automaton AD94 of $A_{1}$. The construction is described below and illustrated by Fig. 9 .

$A_{1,2}$ is now built over the region automaton [AD94 of $A_{1}$. Transitions labeled by some $a \in \mathbb{S}$ are treated separately like in the original construction. The problem now is to constrain $A_{1,2}$ to take one of the most time consuming local runs after a synchronization.

The first step is to build the region automaton of $A_{1}$, and remove the synchronizations. Then, from each state $s$ we compute the most time consuming run and keep only the output arcs of $s$ that start a most time consuming run.

The computation of the most time consuming runs from $s$ is done as follows. If one of the paths from $s$ has a loop, then there is an infinite run from $s$ with local actions, and since we consider non-Zeno TA, time diverges and this run is valid. If no path from $s$ contains a loop, then the paths from $s$ are finite and there is a finite number of such paths. It is possible to compute, for each path, the supremum of the duration of the path: just sum the maximal delays in each location (including the time spent in the last location).

It remains to force, using a fresh clock, the longest stay in each state. 
Lastly, we treat the synchronizations like in the construction of Section 4.1 for each synchronizing edge in $A_{1}$, and each corresponding output state in the region automaton, we add synchronizing edges from all states of $A_{1,2}$, which reset the state of $A_{1,2}$ to the actual state of $A_{1}$. These edges are labeled by " $\gamma(R),\left(a, \ell_{1}\right), c$ ", where $\gamma(R)$ is the constraint that describes the region $R$ associated with the target state, $a$ is the synchronization label in $A_{1}, \ell_{1}$ is the output location of the synchronization in $A_{1}$, and $c$ is the assignment of clock values.

Definition of $A_{1,2}$. Assume $\left(S, s_{0}, E\right)$ is a structure that stores the region automaton of $A_{1}$, without the synchronization edges, and with only the edges that are in the most time consuming paths computed as explained earlier. That is, $S$ (resp. $s_{0}$ ) is the set of states (resp. the initial state) of the region automaton of $A_{1}$, and $E \subseteq S \times\left(\mathbb{N} \times E_{1}\right) \times S$ stores edges in the form $s \stackrel{d, e}{\longrightarrow} s^{\prime}$ where $d$ is the delay that has to be performed in $\ell(s)$, the location associated with state $s$, before performing edge $e$ labeled by some action in $\Sigma_{1} \backslash \mathbb{S}$. Then, $A_{1,2}=\left(S, s_{0}, X_{1} \cup C_{1}^{\prime} \cup\{z\}, \mathbb{S}^{\prime} \cup\{\varepsilon\}, E_{1}^{\prime}, \operatorname{In} v_{1}^{\prime}\right)$ where

- $C_{1}^{\prime}$ is the set of clocks associated with $C_{1}$ as previously, and clocks in $X_{1}$ will be read on the synchronizations only,

- $E_{1}^{\prime}=\left\{s \stackrel{z=d, \varepsilon, r^{\prime} \cup\{z\}}{\longrightarrow} s^{\prime} \mid \exists s \stackrel{d, e}{\longrightarrow} s^{\prime} \in E: e=\left(\ell(s) \stackrel{g, a, c\left(r^{\prime}\right)}{\longrightarrow} \ell\left(s^{\prime}\right)\right)\right\}$

$\cup\left\{s \stackrel{\gamma,\left(a, \ell_{2}\right), c}{\longrightarrow} s^{\prime} \mid s \in S \wedge \gamma \equiv \gamma\left(R\left(s^{\prime}\right)\right) \wedge a \in \mathbb{S} \wedge \exists \ell_{1} \stackrel{g, a, r}{\longrightarrow} \ell_{2} \in E_{1}\right\}$

where $\gamma\left(R\left(s^{\prime}\right)\right)$ is the clock constraint that describes the region of state $s^{\prime}$, and $c$ still denotes the assignment of any clock $x^{\prime} \in C_{1}^{\prime}$ with the value of its associated clock $c\left(x^{\prime}\right)=$ $x \in C_{1}$ (written $x^{\prime}:=x$ ).

- $\forall s \in S, \operatorname{In} v_{1}^{\prime}(s) \equiv z \leq d$ if $\exists s \stackrel{d, e}{\longrightarrow} s^{\prime} \in E$, and $\operatorname{Inv}_{1}^{\prime}(s) \equiv \boldsymbol{t} \boldsymbol{t}$ otherwise.

We can now prove the direct way of Theorem 3.10 in this setting where $A_{1}$ may have urgent synchronizations, and the most time consuming local runs between two synchronizations exist. First, let us recall some notations. $\mathcal{S}_{\text {mod }}=A_{1}^{\prime} \|\left(A_{1,2} \otimes A_{2, \text { mod }}\right)$, with the same $A_{1}^{\prime}$ and $A_{2, \bmod }$ as before, $A_{2}^{\prime}=A_{1,2} \otimes A_{2, \text { mod }}^{\prime}$ where $A_{2, \text { mod }}^{\prime}$ denotes $A_{2, \text { mod }}$ without location $\odot$, and $\psi$ is such that for any $\left(\left(a, \ell_{1}\right), s_{1}\right) \in \mathbb{S}^{\prime} \times Q_{1}^{\prime}, \psi\left(\left(\left(a, \ell_{1}\right), s_{1}\right)\right)=\left(a, s_{1}\right)$.

Proof of Theorem 3.10, when runs of maximal duration before synchronization exist.

We show that when noRestriction $A_{1}\left(A_{2}\right)$ holds, $A_{2}$ does not need to read the clocks of $A_{1}$, because then, the constructed $A_{1}^{\prime} \| A_{2}^{\prime}$ satisfies Definition 3.8, i.e. has no shared clocks and (1) $\psi\left(\operatorname{TTS}_{Q_{1}^{\prime}}\left(A_{1}^{\prime} \| A_{2}^{\prime}\right)\right) \sim \operatorname{TTS}_{Q_{1}}\left(A_{1} \| A_{2}\right)$ and

(2) $\psi\left(\operatorname{TTS}_{Q_{1}^{\prime}}\left(A_{1}^{\prime}\right)\right) \sim \operatorname{TTS}_{Q_{1}}\left(A_{1}\right)$ (this still holds because $A_{1}^{\prime}$ has not changed)

(3) $\psi\left(\operatorname{TTS}_{A_{1}^{\prime}}\left(A_{2}^{\prime}\right)\right) \sim \operatorname{TTS}_{A_{1}}\left(A_{2}\right)$.

First, we can prove that $(*)$ is reachable in $\mathcal{S}_{\text {mod }}$ iff there is a restriction in $\operatorname{TTS}_{A_{1}}\left(A_{2}\right)$, as we proved Lemma 4.2. Indeed, what works when $A_{1,2}$ simulates any run of $A_{1}$ also works when $A_{1,2}$ simulates a fixed run of $A_{1}$.

Then, we can prove that, if $*$ is not reachable (i.e. if there is no restriction in $\operatorname{TTS}_{A_{1}}\left(A_{2}\right)$ ), then $\psi\left(\operatorname{TTS}_{A_{1}^{\prime}}\left(A_{2}^{\prime}\right)\right) \sim \operatorname{TTS}_{A_{1}}\left(A_{2}\right)$. We use the same relation $\mathcal{R}$ as in the previous proof in 4.1. that is, $\mathcal{R}$ is the relation such that for any reachable state $\left(S_{1}, s_{2}\right)$ of $\operatorname{TTS}_{A_{1}}\left(A_{2}\right)$, 

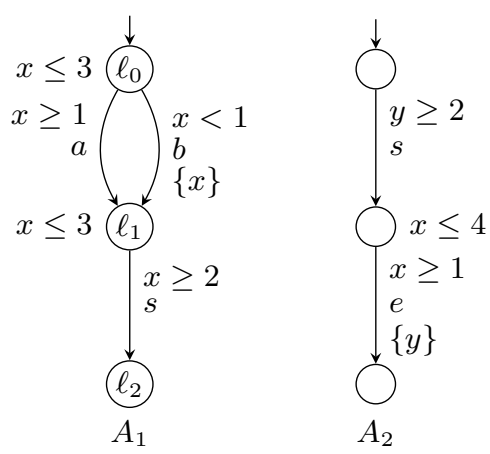

Figure 10: $A_{1}$ has an urgent synchronization and there is no path with maximal duration before this synchronization.

and any reachable state $\left(S_{1}^{\prime}, s_{2}^{\prime}\right)$ of $\psi\left(\operatorname{TTS}_{A_{1}^{\prime}}\left(A_{2}^{\prime}\right)\right)$,

$$
\left(S_{1}, s_{2}\right) \mathcal{R}\left(S_{1}^{\prime}, s_{2}^{\prime}\right) \stackrel{\text { def }}{\Longleftrightarrow}\left\{\begin{array}{l}
s_{2}=\left(\ell_{2}, v_{2}\right) \text { and } s_{2}^{\prime}=\left(\left(\ell_{1,2}, \ell_{2}\right), v_{2}^{\prime}\right) \text { s.t. } \\
\forall x \in X_{2} \backslash X_{1}, v_{2}(x)=v_{2}^{\prime}(x) \\
S_{1}=S_{1}^{\prime}
\end{array}\right.
$$

The proof of this bisimulation follows the same steps as the proof in 4.1, except now we know that $A_{1,2}$ cannot force a synchronization by construction, and not by assuming that there is not urgent synchronization in $A_{1}$.

Then, by Lemma 3.9, $\psi\left(\operatorname{TTS}_{Q_{1}^{\prime}}\left(A_{1}^{\prime} \| A_{2}^{\prime}\right)\right) \sim \operatorname{TTS}_{Q_{1}}\left(A_{1} \| A_{2}\right)$ also.

Case 2: There is not always a Local Run of Maximal Duration after a Synchronization. Now, we show how to adapt the previous construction when there are strict time constraints and there is no path of maximal duration before an urgent synchronization. For example, consider automaton $A_{1}$ of Fig. 10 that has an urgent synchronization and such that there is no path of maximal duration before this synchronization is taken: as previously, $b$ has to be performed as late as possible, but because of the strict inequality $x<1$ on the edge labeled by $b$, it is not possible to enforce this.

Here also, the construction relies on the region automaton and on the computation of the supremum of the possible durations. Then the idea is again to follow one of the paths with the best supremum duration. But there may not exist any optimal timing to run this path and reach the supremum. Then we run it with one possible timing and we wait in the last region, ignoring the invariant that would force us to synchronize. In our example, the supremum of the duration of the path with $b$ is 4 , and is greater than the supremum of any other paths (the paths with $a$ have a maximal duration of 3 ). Therefore, $b$ has to be performed while $x$ is in the region defined by $0<x<1$.

Now, when $A_{1,2}$ reaches a state where it has to synchronize, if $A_{1}^{\prime}$ is not ready to synchronize (i.e. $A_{1}^{\prime}$ is not in the location before the synchronization), then this means that $A_{1}^{\prime}$ took a more time consuming path (and not necessarily the same actions). Then $A_{2, \text { mod }}$ can stop using the values of the clocks of $A_{1,2}$ to evaluate the truth value of its time constraints, and simply take their truth value according to the last region that makes the invariant of the urgent synchronization true (i.e. the region of its current valuation), since it would still be in this region if it had been more time consuming. Note that, if $\odot$ is not 

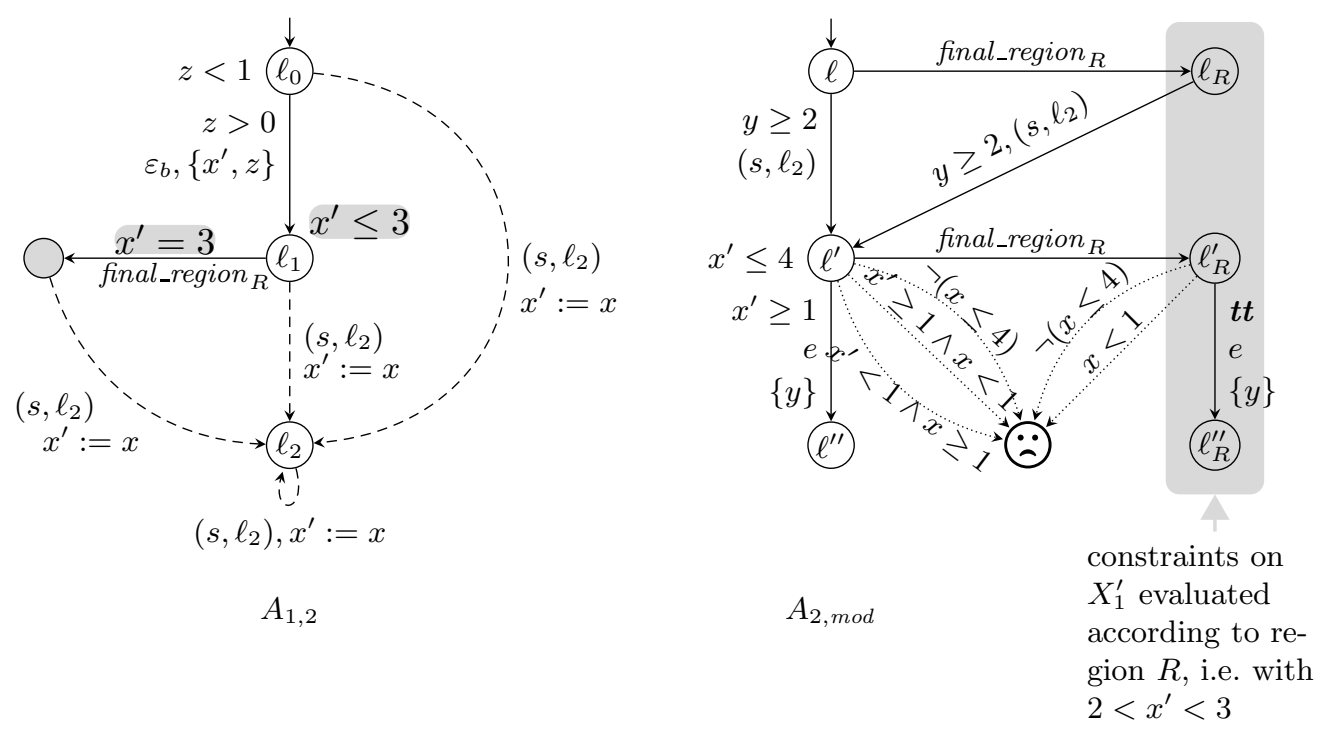

Figure 11: $A_{1,2}$ and $A_{2, \bmod }$ for the NTA of Fig. 10.

reachable, this means that, if $A_{1,2}$ had performed a more time consuming run (for example the actual run followed by $A_{1}^{\prime}$ ), then $A_{2, \text { mod }}$ would have been able to perform the same run. Therefore, "stopping" the clocks in their current region has no side effects.

In the construction, this results in new synchronization edges, performed by $A_{1,2}$ and $A_{2, \text { mod }}$, when $A_{1,2}$ has not been slow enough (i.e. when the invariant expires). In our example, the synchronization labeled by final_region ${ }_{R}$, guarded by $x^{\prime}=3$, notifies $A_{2, \text { mod }}$ that $A_{1,2}$ is stuck in the final region $R$ (here $R$ corresponds to $\ell_{1}$ and $2<x^{\prime}<3$ ) but that its clocks do not satisfy the constraint any more. In this case, $A_{2, \text { mod }}$ enters a duplicated version of itself, where the guards over $X_{1}$ are no more evaluated according to the value of the duplicated clocks $X_{1}^{\prime}$, but simply replaced by their truth value according to the final region. In the example of Fig. 11, the constraint $x^{\prime} \geq 1$ that appears on the arc from $\ell^{\prime}$ to $\ell^{\prime \prime}$ is simply replaced by $\boldsymbol{t} \boldsymbol{t}$, because the constraint is true in region $R$. The duplicated versions can still reach location $\odot$, and the constraints on the edges leading to $\odot$ are also evaluated according to the final region.

If a synchronization happens when $A_{2, \text { mod }}$ is in one of its duplicated versions, then $A_{2, \text { mod }}$ goes back to its initial version, as depicted in Fig. 11.

In order to prove the soundness of the construction, one has to show that if there is no restriction in $\operatorname{TTS}_{A_{1}}\left(A_{2}\right)$ (i.e. if $\odot$ is not reachable), then $\psi\left(\operatorname{TTS}_{A_{1}^{\prime}}\left(A_{2}^{\prime}\right)\right) \sim \operatorname{TTS}_{A_{1}}\left(A_{2}\right)$. The bisimulation relation now takes the new states into account as follows.

$$
\left(S_{1}, s_{2}\right) \mathcal{R}\left(S_{1}^{\prime}, s_{2}^{\prime}\right) \stackrel{\text { def }}{\Longleftrightarrow}\left\{\begin{array}{l}
s_{2}=\left(\ell_{2}, v_{2}\right) \text { and } s_{2}^{\prime}=\left(\left(\ell_{1,2}, \ell_{2}^{\prime}\right), v_{2}^{\prime}\right) \text { s.t. } \\
\ell_{2}=\ell_{2}^{\prime} \text { or } \ell_{2}^{\prime} \text { is one of the duplicated versions of } \ell_{2} \\
\forall x \in X_{2} \backslash X_{1}, v_{2}(x)=v_{2}^{\prime}(x) \\
S_{1}=S_{1}^{\prime}
\end{array}\right.
$$




\section{Discussion And Extensions}

We have shown that in a distributed framework, when locality of actions and synchronizations matter, NTA with shared clocks cannot be easily transformed into NTA without shared clocks. The fact that the transformation is possible can be characterized using the notion of contextual TTS which represent the knowledge of one automaton about the other. Checking whether the transformation is possible is PSPACE-complete.

In system design, our technique could help a designer to use shared clocks in an abstract specification, and build automatically an implementable distributed model without shared clocks. Coming back to the example described in the introduction with several agents performing together a distributed task according to a predefined schedule, this would generate the mechanism for creating the local copies of the schedule.

A first point to notice is that, contrary to what happens when one considers the sequential semantics, NTA with shared clocks are strictly more expressive if we take distribution into account. This somehow justifies why shared clocks were introduced: they are actually more than syntactic sugar.

Another interesting point that we want to recall here is the use of transmitting information during synchronizations. In the end, when the construction is possible, the only modification that is needed for $A_{1}$ is the renaming of the synchronizations, which codes this transmission of information. On the other side, $A_{2}$ needs a much stronger modification in order to handle the information transmitted by $A_{1}$.

Finally, it is noticeable that infinitely precise information is required in general. This advocates the interest of updatable (N)TA used in an appropriate way, and more generally gives a flavor of a class of NTA closer to implementation.

Perspectives. Our first perspective is to generalize our result to the symmetrical case where $A_{1}$ also reads clocks from $A_{2}$. Then of course we can tackle general NTA with more than two automata.

Notice that the set $\operatorname{UR}\left(s_{1}\right)$ used in the definition of contextual TTS is always put in parallel with a state $s_{2}$. Therefore, it can be extended to $\operatorname{UR}_{s_{2}}\left(s_{1}\right)$ that represents the set of states that $A_{1}$ can immediately reach from $s_{1}$ while $A_{2}$ is in $s_{2}$. This means that the TTS of $A_{2}$ in the context of $A_{1}$ can still be defined when $A_{1}$ also reads clocks from $A_{2}$. However, we do not know whether Theorem 3.10 is still true with this definition of contextual TTS, because most of the intermediate lemmas and propositions to prove this theorem use $\operatorname{TTS}\left(A_{1}\right)$ that is not defined when $A_{1}$ reads clocks from $A_{2}$.

Another line of research is to focus on transmission of information. The goal would be to minimize the information transmitted during synchronizations, and see for example where the limits of finite information lay. Even when infinitely precise information is required to achieve the exact semantics of the NTA, it would be interesting to study how this semantics can be approximated using finitely precise information.

Finally, when shared clocks are necessary, one can discuss how to minimize their number, or how to implement the model on a distributed architecture and how to handle shared clocks with as few communications as possible. 


\section{REFERENCES}

$\left[\mathrm{ABG}^{+} 08\right]$ S. Akshay, Benedikt Bollig, Paul Gastin, Madhavan Mukund, and K. Narayan Kumar. Distributed timed automata with independently evolving clocks. In International Conference on Concurrency Theory (CONCUR), volume 5201 of LNCS, pages 82-97, Toronto, Canada, 2008. Springer.

[AD90] Rajeev Alur and David Dill. Automata for modeling real-time systems. In Automata, Languages and Programming, volume 443 of LNCS, pages 322-335. Springer, 1990.

[AD94] Rajeev Alur and David Dill. A theory of timed automata. Theoretical Computer Science, 126(2):183-235, 1994.

[BC12] Sandie Balaguer and Thomas Chatain. Avoiding shared clocks in networks of timed automata. In Maciej Koutny and Irek Ulidowski, editors, Proceedings of the 23rd International Conference on Concurrency Theory (CONCUR'12), volume 7454 of Lecture Notes in Computer Science, Newcastle, UK, September 2012. Springer.

$\left[\mathrm{BCH}^{+} 05\right]$ Béatrice Bérard, Franck Cassez, Serge Haddad, Didier Lime, and Olivier H. Roux. Comparison of the expressiveness of timed automata and time Petri nets. In Paul Pettersson and Wang Yi, editors, FORMATS, volume 3829 of LNCS, pages 211-225. Springer, 2005.

[BCH12] Sandie Balaguer, Thomas Chatain, and Stefan Haar. A concurrency-preserving translation from time Petri nets to networks of timed automata. Formal Methods in System Design, 2012.

[BDFP04] Patricia Bouyer, Catherine Dufourd, Emmanuel Fleury, and Antoine Petit. Updatable timed automata. Theoretical Computer Science, 321(2-3):291-345, 2004.

[BDKP91] Eike Best, Raymond R. Devillers, Astrid Kiehn, and Lucia Pomello. Concurrent bisimulations in Petri nets. Acta Inf., 28(3):231-264, 1991.

[BDL04] Gerd Behrmann, Alexandre David, and Kim Guldstrand Larsen. A tutorial on UPPAAL. In Marco Bernardo and Flavio Corradini, editors, Formal Methods for the Design of Real-Time Systems: 4th International School on Formal Methods for the Design of Computer, Communication, and Software Systems, SFM-RT 2004, number 3185 in LNCS, pages 200-236. Springer-Verlag, September 2004.

$\left[\mathrm{BDM}^{+} 98\right]$ Marius Bozga, Conrado Daws, Oded Maler, Alfredo Olivero, Stavros Tripakis, and Sergio Yovine. KRONOS: a model-checking tool for real-time systems. In $C A V$, volume 1427 of $L N C S$, pages 546550, 1998.

[BDMP03] Patricia Bouyer, Deepak D'Souza, P. Madhusudan, and Antoine Petit. Timed control with partial observability. In Warren A. Hunt, Jr and Fabio Somenzi, editors, CAV 2003, volume 2725 of LNCS, pages 180-192. Springer, Heidelberg, 2003.

[BHR06] Patricia Bouyer, Serge Haddad, and Pierre-Alain Reynier. Timed unfoldings for networks of timed automata. In Susanne Graf and Wenhui Zhang, editors, Proceedings of the 4th International Symposium on Automated Technology for Verification and Analysis (ATVA'06), volume 4218 of LNCS, pages 292-306, Beijing, China, October 2006. Springer.

[BJLY98] Johan Bengtsson, Bengt Jonsson, Johan Lilius, and Wang Yi. Partial order reductions for timed systems. In CONCUR, volume 1466 of $L N C S$, pages 485-500. Springer, 1998.

[BR08] Marc Boyer and Olivier H. Roux. On the compared expressiveness of arc, place and transition time Petri nets. Fundamenta Informaticae, 88(3):225-249, 2008.

[CCJ06] Franck Cassez, Thomas Chatain, and Claude Jard. Symbolic unfoldings for networks of timed automata. In ATVA, volume 4218 of $L N C S$, pages 307-321. Springer, 2006.

[CGL93] Karlis Cerans, Jens Chr. Godskesen, and Kim Guldstrand Larsen. Timed modal specification theory and tools. In Costas Courcoubetis, editor, CAV, volume 697 of LNCS, pages 253-267. Springer, 1993.

[CR06] Franck Cassez and Olivier H. Roux. Structural translation from time Petri nets to timed automata. Journal of Systems and Software, 2006.

[Dim09] Cătălin Dima. Positive and negative results on the decidability of the model-checking problem for an epistemic extension of timed CTL. In TIME, pages 29-36. IEEE Computer Society, 2009.

[DL07] Cătălin Dima and Ruggero Lanotte. Distributed time-asynchronous automata. In ICTAC, pages 185-200. Springer-Verlag, 2007.

[DLLN09] Alexandre David, Kim G. Larsen, Shuhao Li, and Brian Nielsen. Timed testing under partial observability. In ICST, pages 61-70. IEEE Computer Society, 2009. 
[HFMV95] Joseph Y. Halpern, Ronald Fagin, Yoram Moses, and Moshe Y. Vardi. Reasoning About Knowledge. MIT Press, 1995.

[LNZ05] Denis Lugiez, Peter Niebert, and Sarah Zennou. A partial order semantics approach to the clock explosion problem of timed automata. Theoretical Computer Science, 345(1):27-59, 2005.

[LPW07] Alessio Lomuscio, Wojciech Penczek, and Bozena Wozna. Bounded model checking for knowledge and real time. Artif. Intell., 171(16-17):1011-1038, 2007.

[Mer74] Philip Meir Merlin. A study of the recoverability of computing systems. PhD thesis, University of California, Irvine, 1974.

[Min99] Marius Minea. Partial order reduction for model checking of timed automata. In CONCUR, volume 1664 of LNCS, pages 431-446. Springer, 1999.

[Rei84] John Reif. The complexity of two-player games of incomplete information. Jour. Computer and Systems Sciences, 29:274-301, 1984.

[Srb08] Jiří Srba. Comparing the expressiveness of timed automata and timed extensions of Petri nets. In FORMATS, volume 5215 of LNCS, pages 15-32. Springer, 2008.

[vGG01] Rob J. van Glabbeek and Ursula Goltz. Refinement of actions and equivalence notions for concurrent systems. Acta Inf., 37(4/5):229-327, 2001.

[WL04] Bozena Wozna and Alessio Lomuscio. A logic for knowledge, correctness, and real time. In CLIMA, volume 3487 of LNCS, pages 1-15. Springer, 2004. 